\title{
On the Magnitude and Variability of Subgrid-Scale Eddy-Diffusion Coefficients in the Atmospheric Surface Layer
}

\author{
JAN KLEISSL \\ Department of Geography and Environmental Engineering, and Center for Environmental and Applied Fluid Mechanics, \\ The Johns Hopkins University, Baltimore, Maryland \\ Charles Meneveau \\ Department of Mechanical Engineering, and Center for Environmental and Applied Fluid Mechanics, \\ The Johns Hopkins University, Baltimore, Maryland \\ Marc B. Parlange \\ Department of Geography and Environmental Engineering, and Center for Environmental and Applied Fluid Mechanics,
} The Johns Hopkins University, Baltimore, Maryland

(Manuscript received 3 December 2002, in final form 4 April 2003)

ABSTRACT

Eddy-viscosity closures for large eddy simulations (LES) of atmospheric boundary layer dynamics include a parameter (Smagorinsky constant $c_{s}$ ), which depends upon physical parameters, such as distance to the ground, atmospheric stability, and strain. A field study [Horizontal Arrays Turbulence Study (HATS)] specifically designed to measure turbulence quantities of interest in LES, such as the parameter $c_{s}$, is conducted. The instrumentation consists of two vertically separated horizontal arrays of 3D sonic anemometers, placed in the atmospheric surface layer. From 2D filtering and differentiating the velocity fields, subgrid-scale (SGS) and resolved quantities are computed. The parameter $c_{s}$ is obtained from the data by matching measured and modeled SGS dissipations under various flow conditions. Results indicate that $c_{s}$ is reduced near the ground, and also decreases rapidly with increasing stability in stable atmospheric conditions. A simple fit that parameterizes the data is proposed. The variability from one sample to another is studied by means of the probability density function (pdf) of $c_{s}$. The pdfs show a most preferred value, which is essentially independent of the timescale used for statistical averaging. The width of the pdfs decreases with increasing averaging time, for unstable and neutral stability conditions. For stable conditions, the relative variability of the coefficient remains strong even for long averaging times, indicative of strong intermittency. In unstable conditions, $c_{s}$ is fairly independent of local strain-rate magnitude, supporting the basic scaling of the Smagorinsky eddy viscosity. For stable conditions, a transition occurs between small local strain-rate magnitudes, where $c_{s}$ is nearly constant, and high local strain-rate magnitudes, where $c_{s}$ decreases appreciably. The results suggest that when the filter scale approaches the local integral scale of turbulence (height above the ground or Obukhov length), one needs to include the friction velocity as relevant velocity to scale the eddy viscosity, in addition to the standard velocity scale of the Smagorinsky model based on filtered strain-rate magnitude. The analysis is repeated for the SGS heat flux, and for the associated eddy-diffusion coefficient $\left(\operatorname{Pr}_{T}^{-1} c_{s}^{2}\right)$ and Prandtl number $\left(\operatorname{Pr}_{T}\right)$. The latter is found to depend only very weakly on stability, but it increases with decreasing distance from the ground.

\section{Introduction}

Simulations of high Reynolds number turbulent flows, such as the atmospheric boundary layer (ABL), require grid or mesh sizes substantially larger than the smallest scale of motion (the Kolmogorov scale, which is about $\eta \sim 1 \mathrm{~mm}$ in the $\mathrm{ABL}$ ). Large eddy simulation

Corresponding author address: Marc B. Parlange, Dept. of Geography and Environmental Engineering, The Johns Hopkins University, 313 Ames Hall, 3400 N. Charles St., Baltimore, MD 212182686.

E-mail: mbparlange@jhu.edu
(LES; see, e.g., Deardorff 1970; Moeng 1984; Mason 1994; Lesieur and Métais 1996) addresses this problem by resolving the transport equations for all scales of motion larger than the grid size $\Delta$, while the effects of the subgrid scales (SGS) or subfilter scales (SFS; smaller than $\Delta$ ) on the resolved field are parameterized using SGS models. For definitions of SGS and SFS quantities, and a discussion of their differences, see Carati et al. (2001). The realism of the SGS model is essential for the ability of LES to provide realistic turbulent fields in the $\mathrm{ABL}$, especially in regions close to the lower boundary. There the local integral scale is on the order 
of the distance from the boundary $z$. Hence, the SGS model must represent the momentum fluxes carried by most of the eddies, even the large, energy-containing ones. These fluxes are defined using spatial filtering, which separates large and small scales according to

$$
\tilde{\mathbf{u}}(\mathbf{x})=\int \mathbf{u}\left(\mathbf{x}^{\prime}\right) F_{\Delta}\left(\mathbf{x}-\mathbf{x}^{\prime}\right) d \mathbf{x}^{\prime},
$$

where $\tilde{\mathbf{u}}$ is the "resolved" or "filtered" velocity and $F_{\Delta}$ is the (homogeneous) filter function for a scale $\Delta$. In LES, one must model the SGS stress $\tau_{i j}$, defined as

$$
\tau_{i j}=\widetilde{u_{i} u_{j}}-\tilde{u}_{i} \tilde{u}_{j} .
$$

The SGS stresses are three-dimensional, time-dependent, turbulent fields with stochastic character and display a number of interesting statistical properties (for a review, see Meneveau and Katz 2000).

The most commonly employed parameterization for the SGS stress is the Smagorinsky model (Smagorinsky 1963):

$$
\tau_{i j}^{\mathrm{Smag}}-\frac{1}{3} \tau_{k k} \delta_{i j}=-2 \nu_{T} \tilde{S}_{i j}, \quad \nu_{T}=\left(c_{s} \Delta\right)^{2}|\tilde{S}| .
$$

Here, $\tilde{S}_{i j}$ is the strain rate tensor and $|\tilde{S}|=$ $\sqrt{2 \tilde{S}_{i j} \tilde{S}_{i j}}$ is its magnitude; $\nu_{T}$ is the eddy viscosity; and $c_{s}$ is the Smagorinsky coefficient, which, in traditional LES, is prescribed based on phenomenological theories of turbulence or adjusted empirically. The product of Smagorinsky coefficient and filter scale is a mixing length often denoted by $l=\Delta c_{s}$. For a recent review of the Smagorinsky model and other SGS models, see Meneveau and Katz (2000). As also reviewed in this reference, the magnitude of $c_{s}$ determines the effectiveness with which kinetic energy is dissipated out of the resolved velocity field during LES. The mean rate of kinetic energy transfer from the resolved to the subgrid range of scales (the so-called SGS dissipation) is given by

$$
\left\langle\Pi^{\text {meas }}\right\rangle=-\left\langle\tau_{i j} \tilde{S}_{i j}\right\rangle,
$$

where \langle\rangle denotes ensemble or time averaging, depending on the context. The rate that results from replacing $\tau_{i j}$ with the Smagorinsky model is given by

$$
\left\langle\Pi^{\text {Smag }}\right\rangle=2\left(c_{s} \Delta\right)^{2}\left\langle|\tilde{S}| \tilde{S}_{i j} \tilde{S}_{i j}\right\rangle .
$$

By requiring that $\left\langle\Pi^{\text {meas }}\right\rangle=\epsilon=\left\langle\Pi^{\text {Smag }}\right\rangle$ (where $\epsilon$ is the molecular dissipation rate), Lilly (1967) analytically derives a value of $c_{s}$ of approximately $0.16-0.20$ (the exact value depends on the filter shape and the Kolmogorov constant). His main assumption is the application of a filter operation at a scale $\Delta$ that falls within an idealized inertial subrange of turbulence with energy spectrum $E(k)=\alpha \epsilon^{2 / 3} k^{-5 / 3}$ to evaluate $\left\langle|\tilde{S}| \tilde{S}_{i j} \tilde{S}_{i j}\right\rangle$. This derived value of $c_{s}$ exceeds significantly what LES calculations require to yield realistic results, especially close to the ground (Deardorff 1970; Moin and Kim 1982; Mason and Thomson 1992; Sullivan et al. 1994). As is widely recognized, near the ground, $\Delta$ approaches or exceeds energy-containing scales, and hence the basic assumption of Lilly (1967) breaks down.

Wall-blocking effects are known to cause a reduction in the coefficient when approaching the ground. Mason (1994) proposes to match the basic mixing length of the Smagorinsky model in the interior of the ABL, $l_{0}=$ $c_{0} \Delta$, with rough-surface expressions for the eddy viscosity $\nu_{T}=\kappa^{2}\left(z+z_{0}\right)^{2} \partial\langle u\rangle / \partial z$ near the ground. Mason's (1994) modified mixing length $l$ reads

$$
l=\left\{\frac{1}{\left[\kappa\left(z+z_{0}\right)\right]^{n}}+\frac{1}{l_{0}^{n}}\right\}^{-1 / n} .
$$

Thermal stratification also influences the SGS energy spectrum of turbulence, which in turn violates Lilly's assumption of a long inertial subrange in deriving $c_{s}$. In particular, the coefficient has to be decreased in stably stratified conditions. This trend is reflected in Deardorff's (1980) empirical model, as well as in the model of Brown et al. (1994), who derive a stability-dependent model from the SGS energy equation assuming a state of local equilibrium. Canuto and Cheng (1997) employ a two-point closure to construct the SGS energy spectrum under the influence of shear and buoyancy. From the SGS energy spectrum they derive an analytical expression for the reduction of $c_{s}$ under shear and buoyancy. Like stratification, the presence of mean shear also requires decreasing the Smagorinsky coefficient.

Another approach, quite different from using analytical expressions that model the postulated dependence of $c_{s}$ as a function of flow parameters, is the so-called dynamic model of Germano et al. (1991). In the dynamic model the resolved turbulence at scales between $\Delta$ and $2 \Delta$ is analyzed statistically during the simulation, and coefficients are deduced from appropriate averaging operations. There is considerable evidence that the dynamic model is able to provide realistic predictions of the coefficient, at least when the filter scale $\Delta$ and the test-filter scale $2 \Delta$ are smaller than the local integral scale of turbulence. Porté-Agel et al. (2000a) generalize the dynamic model to include scale dependence and show successful application to a neutral boundary layer.

Regardless of which approach is used to model the coefficient value, reliable information is needed about what is the correct value of $c_{s}$ under different flow conditions. In this paper we use data from field experiments to measure $c_{s}$ under flow conditions prevalent in the atmospheric surface layer. In order to measure $c_{s}$ under flow conditions that are more general than the isotropic conditions of Lilly's (1967) original derivation, his theoretical approach can be applied to analysis of experimental data by setting the dissipation from the Smagorinsky model equal to the real measured SGS dissipation, that is, by setting $\left\langle\Pi^{\text {meas }}\right\rangle=\left\langle\Pi^{\text {smag }}\right\rangle$. An empirically measurable SGS dissipation-based Smagorinsky coefficient can thus be defined as follows: 


$$
c_{s}^{2}=-\frac{\left\langle\tau_{i j} \tilde{S}_{i j}\right\rangle}{\left\langle 2 \Delta^{2}|\tilde{S}| \tilde{S}_{i j} \tilde{S}_{i j}\right\rangle} .
$$

This approach was pioneered by Clark et al. (1979) for the analysis of data from Direct Numerical Simulations (DNS). As reviewed in Meneveau and Katz (2000), since then many studies have used this criterion to compute $c_{s}$.

In this paper we aim to derive, from the field data, empirical relationships for $c_{s}$ as a function of relevant parameters such as distance to the ground, strength of thermal stratification, and strain-rate magnitude. The distance to the ground $z$ can be normalized with the filter scale $\Delta$ yielding the parameter $\Delta / z$. Stratification can be characterized using the Obukhov length $L$, defined as

$$
L=\frac{-u_{*}^{3} \rho}{\kappa g\left(\frac{H}{C_{p} \theta_{0}}+0.61 E\right)} \approx \frac{-u_{*}^{3}}{\kappa \frac{g}{\theta_{0}}\left\langle w^{\prime} \theta^{\prime}\right\rangle} .
$$

Here, $u_{*}=\left(-\left\langle u^{\prime} w^{\prime}\right\rangle\right)^{1 / 2}$ is the friction velocity, $\rho$ is the density of air, $H$ is the sensible heat flux, $\theta_{0}$ is the mean air temperature, $E$ is the water vapor flux, $g$ is the gravitational acceleration and $\kappa=0.4$ is the von Kármán constant. The dimensionless parameter comparing the filter scale to $L$ is $\Delta / L$. The local strain rate will be quantified by $|\tilde{S}|$, the magnitude of the strain-rate tensor already defined in Eq. (3). It can be normalized with a velocity scale and a length scale. The proper choice of velocity and length scales depends on whether $\Delta$ falls inside or outside the inertial range.

In addition to the dependence of $c_{s}$ on these parameters, the great variability of turbulence dynamics in general, and of atmospheric dynamics in particular, raises the issue of how the averaging procedures needed in evaluating terms in Eq. (7) should be performed, and how meaningful the results are. Variability in $c_{s}$ is caused by the inherent intermittency of turbulence, and of ABL flow patterns in particular. It is well known that the SGS dissipation $\Pi^{\text {meas }}$ in turbulence is highly intermittent. This was already shown for isotropic turbulence using DNS by Cerutti and Meneveau (1998) and for the ABL in the context of the SGS dissipation of scalar variance by previous experiments described in PortéAgel et al. (2000b, 2001a,b). To examine the effects of intermittency upon eddy-viscosity coefficients, the averages in the nominator and denominator of Eq. (7) can be computed over different timescales $T_{c}$. Then $c_{s}$ is no longer a single value but fluctuates from one time period (of length $T_{c}$ ) to another. We wish to examine how this variability is affected by varying $T_{c}$ under different flow conditions. Moreover, in LES using the Lagrangian dynamic model (Meneveau et al. 1996), one needs to prescribe a timescale. This timescale is used in that model to set the duration of averaging over the history of turbulence following fluid trajectories.

Similarly to the filtered momentum equations, the fil- tered scalar transport equations (e.g., heat equation) in LES include an additional term, the SGS scalar fluxes. The SGS heat flux is defined according to

$$
q_{i}=\widetilde{\theta u_{j}}-\tilde{\theta} \tilde{u}_{j},
$$

where $\theta$ is the temperature field. In the Smagorinsky, or eddy-diffusivity model, $q_{i}$ is parameterized as

$$
q_{i}^{\text {Smag }}=-\operatorname{Pr}_{T}^{-1} c_{s}^{2} \Delta^{2}|\tilde{S}| \frac{\partial \tilde{\theta}^{\prime}}{\partial x_{i}},
$$

where $\operatorname{Pr}_{T}$ is the turbulent SGS Prandtl number, and $\tilde{\theta}$ is the filtered temperature field. The prime indicates fluctuating quantities around the average $(\tilde{\theta}=\langle\tilde{\theta}\rangle+$ $\left.\tilde{\theta}^{\prime}\right)$. The mean SGS dissipation of scalar variance $\left\langle\chi^{\text {meas }}\right\rangle$ is usually defined as

$$
\left\langle\chi^{\text {meas }}\right\rangle=-\left\langle q_{i} \frac{\partial \tilde{\theta}^{\prime}}{\partial x_{i}}\right\rangle .
$$

Lilly's analysis applied to a scalar variance spectrum in isotropic, neutral turbulence led to an estimate of the Prandtl number of about 0.5 for the Smagorinsky model (Mason 1994). Laboratory experimental investigations in the wake of a heated cylinder (Kang and Meneveau 2002) resulted in $\operatorname{Pr}_{T} \approx 0.3$. From experimental data, the scalar eddy-diffusion coefficient can be determined using the criterion that the mean modeled SGS dissipation of scalar variance $\left\langle\chi^{\text {mod }}\right\rangle=-\left\langle q_{i}^{\text {Smag }} \partial \tilde{\theta}^{\prime} / \partial x_{i}\right\rangle$ matches the mean measured SGS dissipation of scalar variance $\left\langle\chi^{\text {meas }}\right\rangle$ :

$$
\operatorname{Pr}_{T}^{-1} c_{s}^{2}=\frac{-\left\langle q_{i} \frac{\partial \tilde{\theta}^{\prime}}{\partial x_{i}}\right\rangle}{\left\langle\Delta^{2}|\tilde{S}| \frac{\partial \tilde{\theta}^{\prime}}{\partial x_{i}} \frac{\partial \tilde{\theta}^{\prime}}{\partial x_{i}}\right\rangle} .
$$

The Prandtl number can be obtained by dividing the result for $c_{s}^{2}$ from Eq. (7) by $\operatorname{Pr}_{T}^{-1} c_{S}^{2}$. As part of the present study we will also examine how $\operatorname{Pr}_{T}^{-1} c_{s}^{2}$ (and $\operatorname{Pr}_{T}$ ) depend on distance to the ground, flow stability, and averaging timescale.

Before proceeding to the details of the present data and analysis, it is worthwhile to delineate two aspects of the deliberately focussed scope of the present study: First, we restrict attention to the basic structure of the eddy-viscosity Smagorinsky closure. This closure is based on the assumption that the SGS stresses and fluxes are aligned to the gradients of velocity and temperatures. The drawbacks of this assumption have already been documented extensively in the literature. As reviewed in Meneveau and Katz (2000) and Tao et al. (2002) in the context of experimental studies in laboratory turbulence, the alignment hypothesis is not accurate. In the context of ABL turbulence, Higgins et al. (2003) confirm this limitation and show that addition of a so-called tensor eddy-diffusion model improves the alignment trends. Moreover, near the ground, Tong et al. (1999) show that the streamwise accelerations inherent in the 
eddy-viscosity closures cause unphysical couplings with the resolved velocity field. Even with these limitations, the eddy-viscosity closure is still the most often used in practical applications, providing continued interest in the dependence of $c_{s}$ on physical flow parameters as studied here.

Second, in this work the coefficient is measured based only on the condition of SGS energy and scalar variance dissipation equivalence [Eqs. (7) and (12)]. While it is often argued that this is the most important condition (Meneveau and Katz 2000), we recall that accurate prediction of SGS dissipation is only one of many possible conditions with which an SGS model should comply. As enumerated in Meneveau (1994) and Pope (2000, p. 603) several other statistics are of interest, such as dissipation of enstrophy, or wavenumber-dependent spectral transfer leading to spectral eddy viscosity (Cerutti et al. 2000). In fact, in the context of near-surface ABL flows where the SGS stress carries a significant fraction of the total vertical fluxes of momentum, an additional condition could be that the modeled SGS shear stress equals the real one. An alternative definition of the Smagorinsky coefficient, named $c_{s}^{\text {mom }}$, which satisfies the condition of equivalence of vertical fluxes of momentum would read

$$
\left(c_{s}^{\text {mom }}\right)^{2}=-\frac{\left\langle\tau_{13}\right\rangle}{\left\langle 2 \Delta^{2}|\tilde{S}| \tilde{S}_{13}\right\rangle},
$$

where $x_{1}=x$ and $x_{3}=z$ are streamwise and vertical directions, respectively. How to combine this condition with the energy-based condition of Eq. (7), and how to address the problem that Eq. (13) becomes ill posed when $\Delta / z \ll 1$ [there the numerator and denominator of Eq. (13) become negligible], are questions that require significant attention beyond the scope of the present study.

The present paper is organized as follows. In section 2, we describe the field experiment and the dataset used in the present study. Section 3 presents results on the magnitude of the measured $c_{s}$ as function of atmospheric stability and distance to the ground. An empirical fit to represent the dependence of $c_{s}$ on the flow parameters for easy use in the context of LES is presented. Section 4 studies the dependence of $c_{s}$ on the local strain-rate magnitude. Section 5 examines in more detail the issue of statistical variability of $c_{s}$, as function of averaging timescale and atmospheric stability. Section 6 shows results from a similar analysis for the SGS heat flux. Conclusions are presented in section 7.

\section{The Horizontal Array Turbulence Study (HATS) dataset}

In the context of LES for the atmospheric boundary layer, a number of field studies have aimed at measuring $q_{i}$ and $\tau_{i j}$ from field data and at analyzing the results to improve SGS modeling. A study using data from a single 3D sonic anemometer (Porté-Agel et al. 1998) re- stricted the analysis to one-dimensional filtering (time filtering and interpreting the results as filtering in the $x_{1}$ $=x$ direction using Taylor's hypothesis), whereas the filtering assumed in Eq. (1) is in all three directions. Henceforth, $x_{1}=x, x_{2}=y$, and $x_{3}=z$ will be used interchangeably. Tong et al. (1998) proposed deploying a horizontal array of sensors and examined filtering issues using LES-data. Their results showed that filtering in two horizontal directions was required for quantitatively more accurate results. Experimental results from one horizontal array of sensors using two-dimensional filtering were reported in Tong et al. (1999) and PortéAgel et al. (2000b). The latter paper showed that, while filter dimensionality did not have a strong effect on the previously reported trends based on one-dimensional filtering, atmospheric stability had strong effects on the results. Limiting the setup of Porté-Agel et al. (2000b) was the inability to compute vertical derivatives. This issue was addressed by using two vertically displaced horizontal arrays as proposed in Tong et al. (1999), and also in the Davis 1999 experiment (Porté-Agel et al. 2001a). As described in the next paragraphs, a similar setup is used in the present study [Horizontal Array Turbulence Study (HATS)] now including two more anemometers, and including more data under stable stratification, due to prevailing wind conditions at night.

HATS was conducted in the San Joaquin Valley close to Kettleman City, California, from 31 August until 1 October 2000. The field site was selected because of its homogeneous surface conditions with predictable wind directions. It was located $5.6 \mathrm{~km}$ east-northeast of Kettleman City at the southeast corner of an area of unplanted farmland. Homogeneous surface conditions ranged at least $2 \mathrm{~km}$ in the upwind (northwest) direction. Vegetation consisted of crop stubble and weeds for which the displacement height $d_{0}$ and roughness length $z_{0}$ were calculated to be 32 and $2 \mathrm{~cm}$, respectively. As outlined in the introduction the goal of the experiment was the examination of SGS quantities for a wide range of stabilities $\Delta / L$ and array geometries $\Delta / z$. The requirement of computing derivatives in all directions necessitated a setup of 3D sonic anemometers in two parallel horizontal arrays, which are separated in the vertical direction and centered in the lateral direction (see Fig. 1). Variation in $\Delta / z$ was achieved by selecting four setups with different geometrical arrangements (see Table 1), each of which was in the field for 6-9 days with continuous sampling in order to record data for a wide range of stabilities $\Delta / L$. A total of 14 Campbell Scientific three-component sonic anemometer-thermometers (CSAT3) were partitioned into one array with nine sonics and another array with five instruments. The former allows for computation of double-filtered quantities and is named the subscript " $d$ " array, while the latter is referred to as subscript " $\mathrm{s}$ " array as in single filtered. An additional two sonics were mounted on a reference tower to examine flow obstruction. For additional in- 


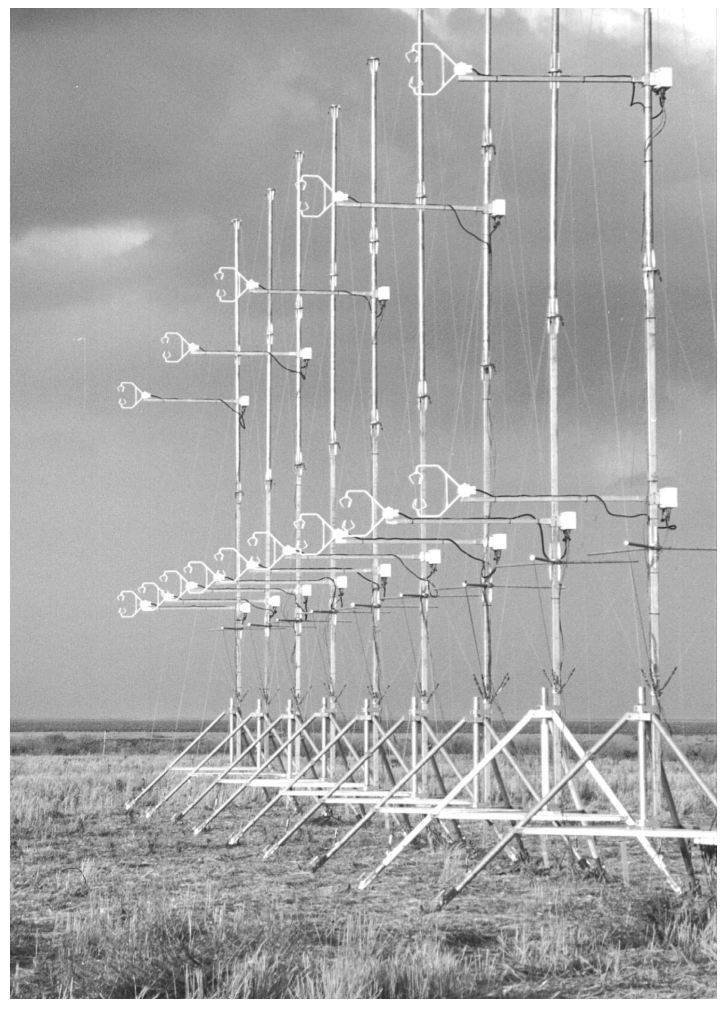

FIG. 1. Photograph of the setup of array 1 during the HATS experiment near Kettlamen City, California. Photo courtesy of Tom Horst, NCAR.

formation see Horst et al. (2003, unpublished manuscript).

All 16 sonics were calibrated before and after the experiment in the National Center for Atmospheric Research (NCAR) wind tunnel and differences in the slope of regressions for the 16 sonics were in a range on the order of $2 \%$. The standard deviation of the slope of the regressions was less than $0.5 \%$. All sonics met the specification of the manufacturer of an intercept of less than $4 \mathrm{~cm} \mathrm{~s}^{-1}$, only one had an offset of $6 \mathrm{~cm} \mathrm{~s}^{-1}$ after the experiment. Other errors stem from the alignment of the sonic anemometers. Errors in the alignment of the $x-y$ plane of the sonic anemometers parallel to the surface can be corrected for in postprocessing assuming that the mean wind vector is parallel to the local surface. This tilt was found to be less than $2^{\circ}$. The $x$ axes of all sonics should be parallel to each other and perpendicular to the $x-z$ array plane. The error in this alignment was measured onsite with a theodolite. After correcting the data with the theodolite measurements intercomparisons of horizontal wind components of the instruments still showed offsets of up to $6 \mathrm{~cm} \mathrm{~s}^{-1}$ and residual wind direction biases of up to $2^{\circ}$. This paragraph summarizes the descriptions in Horst et al. (2003, unpublished manuscript), where a more detailed data quality analysis is presented.

The temperature measurements are uncalibrated. However, the present analysis does not involve any vertical gradients of mean temperature, but only gradients of temperature fluctuations. By subtracting the mean temperature from each instrument's measurement, the remaining error is due to experimental uncertainty in the output. It is specified by the manufacturer as $0.026 \mathrm{~K}$.

The arrays were oriented in a way that southeastward winds $\left(315^{\circ}\right)$ were perpendicular to the arrays and caused the least interinstrumental flow obstruction. For our analysis, all time periods with an angle of the downstream pointing array normal and 6.8-min averaged wind vector of $-30^{\circ}<\alpha<30^{\circ}$ are considered. Excluding all data violating this criterion leaves us with the amount of data specified in the second column of Table 1. During data processing, the array is rotated to a position perpendicular to the prevailing wind using Taylor's hypothesis. The center of rotation for both arrays is the center sonic (same $y$ coordinate). The new (rotated) velocity for a sonic with distance $\delta_{y}$ from the center sonic for given mean horizontal velocity vector $\langle u\rangle$ and angle of average wind vector with the array normal $\alpha$ is $u_{i}^{\text {new }}(x, y, z, t)=u_{i}\left(x, y, z, t-\delta_{y} \sin \alpha /\langle u\rangle\right)$. This rotation results in a decrease of the effective filter size to $\Delta_{\text {eff }}=\Delta \cos \alpha$. For the remainder of the paper, all statements involving filter size refer to the effective filter size. Sonic anemometer signals were sampled at a data acquisition frequency of $20 \mathrm{~Hz}$.

Filtered quantities that were defined as a continuum in Eq. (1) have to be computed using discrete filters as specified in Table 1. Many LES codes use a 2D spectral cutoff filter in horizontal planes. However, this filter is not suited for our analysis, because its slow $x^{-1}$ decay in physical space aggravates its approximation with $O(5)$ sensors. Moreover the spatial cutoff filter produces a spatially nonlocal impact when filtering spatially lo-

TABLE 1. Array properties for the HATS experiment: "d" is double-filtered array; "s" is single-filtered array; $d_{0}$ is displacement height; $\Delta$ is filter size. The last three columns specify the type of filter used in the $x$ and $y$ directions. The number following the filter type specifies the number of instruments over which the spatial average is computed. Note that, for the remainder of the paper, the data for arrays 3 and 4 are merged, because their $z / \Delta$ values are similar.

\begin{tabular}{|c|c|c|c|c|c|c|c|c|c|}
\hline $\begin{array}{c}\text { Array } \\
\text { (no.) }\end{array}$ & Data (h) & $z_{d}-d_{0}(\mathrm{~m})$ & $z_{s}-d_{0}(\mathrm{~m})$ & $\Delta(\mathrm{m})$ & $\frac{\Delta}{z_{d}-d_{0}}(-)$ & $\left\langle u_{d}\right\rangle\left(\mathrm{m} \mathrm{s}^{-1}\right)$ & $x$ filter & $y$ filter $_{d}$ & $y$ filter $_{s}$ \\
\hline 1 & 46.0 & 3.13 & 6.58 & 13.4 & 4.28 & 2.46 & Gaussian & Trapezoidal, 5 & Trapezoidal, 3 \\
\hline 2 & 38.7 & 4.01 & 8.34 & 8.68 & 2.16 & 2.72 & Gaussian & Trapezoidal, 5 & Trapezoidal, 3 \\
\hline 3 & 37.9 & 8.34 & 4.01 & 4.34 & 0.52 & 2.25 & Gaussian & Trapezoidal, 3 & Trapezoidal, 5 \\
\hline 4 & 34.5 & 3.83 & 4.83 & 2.50 & 0.65 & 2.41 & Gaussian & Top-hat, 4 & Trapezoidal, 5 \\
\hline
\end{tabular}


calized phenomena ("ringing"). Thus, we choose to use spatially localized filters, which can be well represented by the experimental arrangement. In the lateral $(y)$ direction trapezoidal filter functions are used with the exception of array 4, for which a top-hat filter is used for the $d$ array in order to match the filter sizes of $s$ and $d$ arrays. For increased smoothness Gaussian filter functions are applied in the streamwise $(x)$ direction where a higher resolution is available due to the $20-\mathrm{Hz}$ sampling that corresponds to a sampling distance of about $0.12 \mathrm{~m}$, using Taylor's hypothesis. Filtering is done in wave space using the Fourier transform of the Gaussian filter function $\hat{G}_{\Delta}=\exp \left[-\left(k_{1}^{2} \Delta^{2} / 24\right)\right]$, where $k_{1}$ is the wavenumber. Cerutti and Meneveau (2000) confirmed the feasibility of a box filter for spatially averaging a finite number of sensors; Porté-Agel et al. (2001a) concluded that their results for $c_{s}$ were not strongly affected by the choice of streamwise filter function.

Gradients are calculated in all directions using finite differences (FD). For gradients in the vertical direction $\left(x_{3}=z\right)$ a first-order one-sided FD over a distance $\left(z_{s}\right.$ $\left.-z_{d}\right)$ is imposed by the geometry $\left(\partial \tilde{u} /\left.\partial z\right|_{z_{d}}=\left(z_{s}-\right.\right.$ $\left.\left.z_{d}\right)^{-1}\left[\tilde{u}\left(z_{s}\right)-\tilde{u}\left(z_{d}\right)\right]\right)$. In the horizontal directions a fourth-order centered FD scheme is applied; that is,

$$
\begin{aligned}
\left.\frac{\partial \widetilde{u}_{i}}{\partial y}\right|_{y_{0}}=\frac{1}{12 \delta_{y}}[ & \widetilde{u}_{i}\left(y_{0}+2 \delta_{y}\right)+8 \widetilde{u}_{i}\left(y_{0}+\delta_{y}\right) \\
& \left.-8 \widetilde{u}_{i}\left(y_{0}-\delta_{y}\right)-\widetilde{u}_{i}\left(y_{0}-2 \delta_{y}\right)\right],
\end{aligned}
$$

for the $y$ direction, where $\delta_{y}$ is the lateral spacing of the sonic anemometers. Equation (14) with $\delta_{x}=\delta_{y}$ is used in the streamwise direction for computing spatial derivatives from time derivatives using Taylor's hypothesis $\partial / \partial x=-\langle u\rangle \partial / \partial t$. Since the accuracy of spatial gradients is important for the analysis of modeled quantities and coefficients [e.g., Eqs. (7) and (12)], they are examined in more detail in the appendix.

\section{Dependence of $c_{s}$ on stability and height}

In order to study the effect of stability and height, the data are divided into segments of length $T_{L}$ (we mostly use $T_{L}=6.8$-min-long segments containing $2^{13}$ points), that are classified in terms of Obukhov length $L$ [defined according to Eq. (8)], and height $\Delta / z$. To illustrate the total amount of data, the cumulative duration of all segments in each $\Delta / L$ bin and $\Delta / z$ bin is shown in Fig. 2. As can be seen, more data are available in the near-neutral bins while less data are available in the more stable bins. There are $\sim 40 \mathrm{~h}$ of useful data for each array, which implies that there is more data available for the $\Delta / z<0.7$ case, because data from arrays 3 and 4 are combined in this bin. As outlined in the introduction, in the paper various averaging timescales $T_{c}$ will be used to compute $c_{s}$ from Eq. (7).

We begin by analyzing data from array 2 , with $\Delta / z$

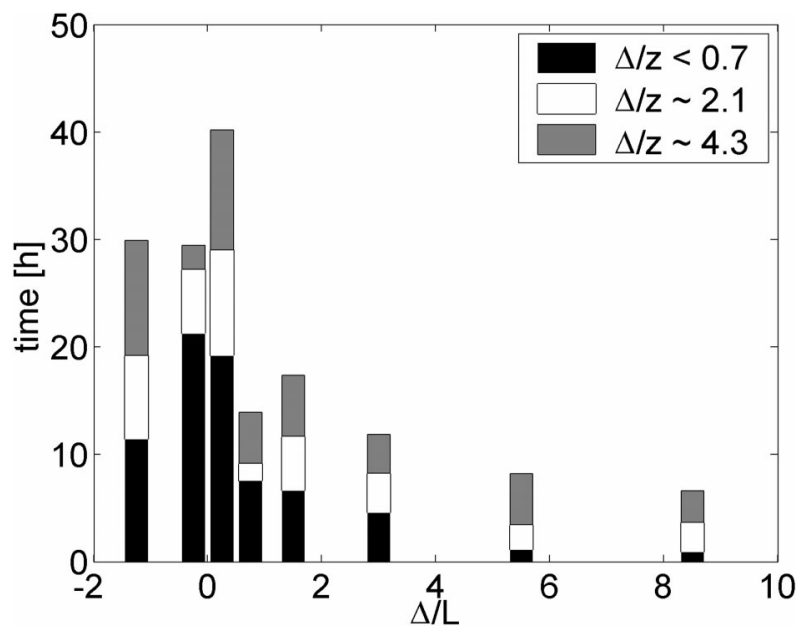

FIG. 2. Cumulative time of available data in each data bin. All 6.8min data segments whose average horizontal wind vector is less than $30^{\circ}$ off the array normal are binned according to their $\Delta / L$ and $\Delta / z$ value. The height range $(\Delta / z)$ is partitioned into three bins: array 1 $(\Delta / z \sim 4.3)$, array $2(\Delta / z \sim 2.1)$, and arrays 3 and 4 , which are combined $(\Delta / z<0.7)$. The stability range $(\Delta / L)$ is partitioned into eight bins, whose end points are given by the list $[-2.0,-0.5,0$, $0.5,1.0,2.0,4.0,7.0,10.0]$.

$\sim 2.1$ (i.e., $\Delta \sim 8.6 \mathrm{~m}$ ), and dividing the data into short subsegments of length $T_{c}=3.2 \mathrm{~s}$. With a representative mean velocity of $\langle u\rangle \sim 2.72 \mathrm{~m} \mathrm{~s}^{-1}$ this timescale corresponds to a length scale $T_{c}\langle u\rangle \sim 8.7 \mathrm{~m}$, that is, on the order of the filter scale $\Delta \sim 8.6 \mathrm{~m}$. We consider data with $\Delta / L$ ranging between -3.0 and 11.5 . We then proceed to compute the Smagorinsky model coefficient according to Eq. (7) by evaluating the averages over time $T_{c}$ and classifying the result according to the value of $\Delta / L$. Due to the smaller averaging timescale $\left(T_{c}=3.2\right.$ s) compared to Fig. 2, more values of $c_{s}$ are available and a finer bin resolution for $\Delta / L$ is chosen (18 bins). Even for a fixed $\Delta / L$ the resulting $c_{s}$ displays considerable variability from one sample to another. Thus, we compute the conditional pdf of $c_{s}^{2}$, defined in terms of the joint pdf $P\left(c_{s}^{2}, \Delta / L\right)$ according to

$$
P\left(c_{s}^{2} \mid \frac{\Delta}{L}\right)=\frac{P\left(c_{s}^{2}, \frac{\Delta}{L}\right)}{P\left(\frac{\Delta}{L}\right)},
$$

where $P(\Delta / L)$ is the fraction of data contained in each $\Delta / L$ bin. In this fashion the dependence on $\Delta / L$ is isolated, independent of the amount of data in different stability bins in our dataset [there is much more nearneutral data than stably stratified data, which biases the joint pdf $P\left(c_{s}^{2}, \Delta / L\right)$ toward low values of $\left.\Delta / L\right]$. To construct the pdf, the range of $c_{s}^{2}\left(-0.02<c_{s}^{2}<0.04\right)$ is divided into 120 bins. The resulting conditional pdf of the coefficient is shown using color contours in Fig. 3a for the $c_{s}^{2}$ and $\Delta / L$ range, where sufficient data are avail- 

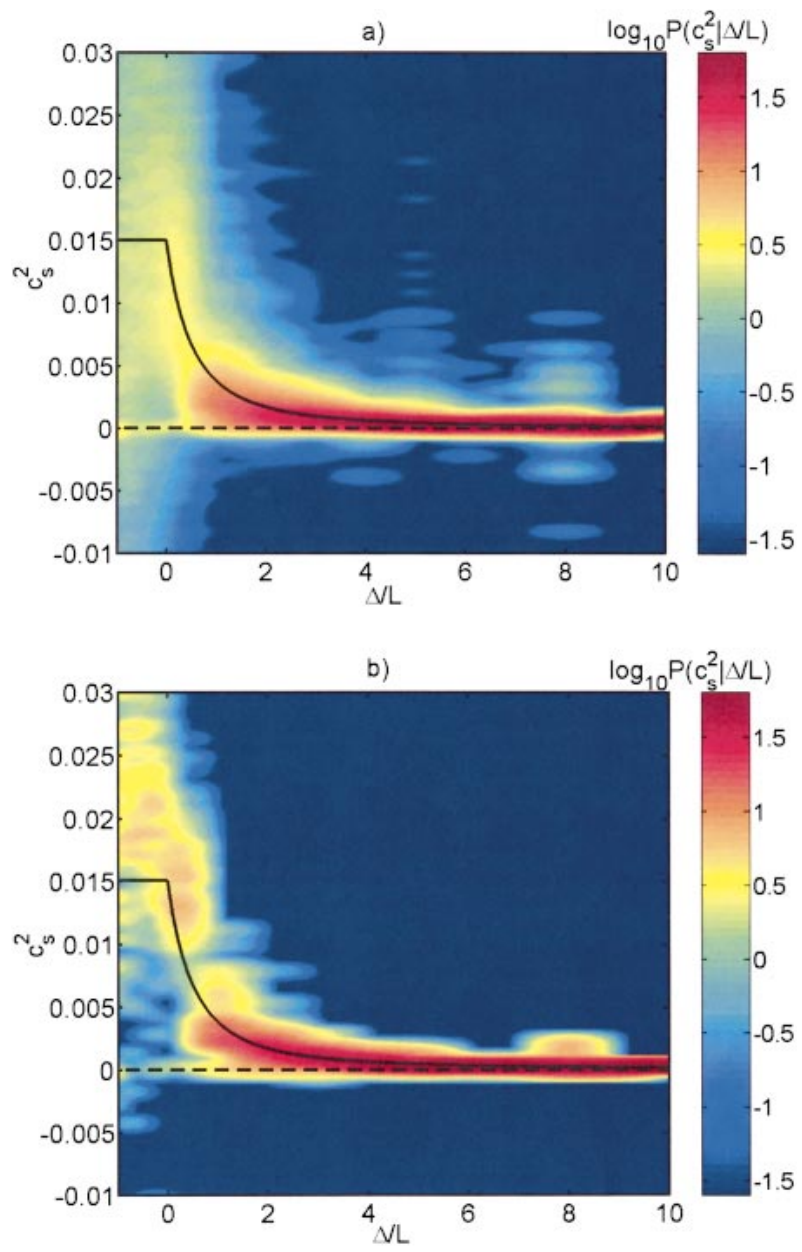

FIG. 3. Contour plots of conditional pdf of $c_{s}^{2}, P\left(c_{s}^{2} \mid \Delta / L\right)$. The contours are spaced logarithmically. (a) The averaging time to compute $c_{s}^{2}$ is $T_{c}=3.2 \mathrm{~s} \sim 1.0 \Delta /\langle u\rangle$; the averaging time to compute $c_{s}^{2}$ (b) is $T_{c}=102.4 \mathrm{~s} \sim 32 \Delta /\langle u\rangle$. Results are from array 2 with $\Delta / z \sim$ 2.1. The solid line is an empirical fit described in Eq. (17). The dashed line shows $c_{s}^{2}=0$.

able. Repeating the procedure for a longer averaging time $T_{c}=102.4 \mathrm{~s}$, corresponding to about $32 \Delta /\langle u\rangle$, we obtain the conditional pdf shown in Fig. $3 b$.

Figure $3 \mathrm{a}$ shows that the most likely value of $c_{s}^{2}$ depends strongly on stability. Specifically, $c_{s}^{2}$ decreases from values fluctuating around $\sim 0.015$ in neutral conditions to smaller values for increasing $\Delta / L$. The parameter $c_{s}^{2}$ is particularly sensitive to stability in the slightly stable region $0<\Delta / L<0.5$. For unstable conditions, there is a large spread in $c_{s}^{2}$ values around its conditional mean value, whereas for very stable conditions all $c_{s}^{2}$ fall within a narrower range. For unstable conditions there is a significant amount of negative $c_{s}^{2}$. These events are called backscatter events, because the resulting negative eddy viscosity causes an energy transfer from the SGS to the resolved scales during the time period $T_{c}$. When the averaging time $T_{c}$ for the computation of $c_{s}^{2}$ is increased (Fig. 3b), the spread in $c_{s}^{2}$ decreases sig-

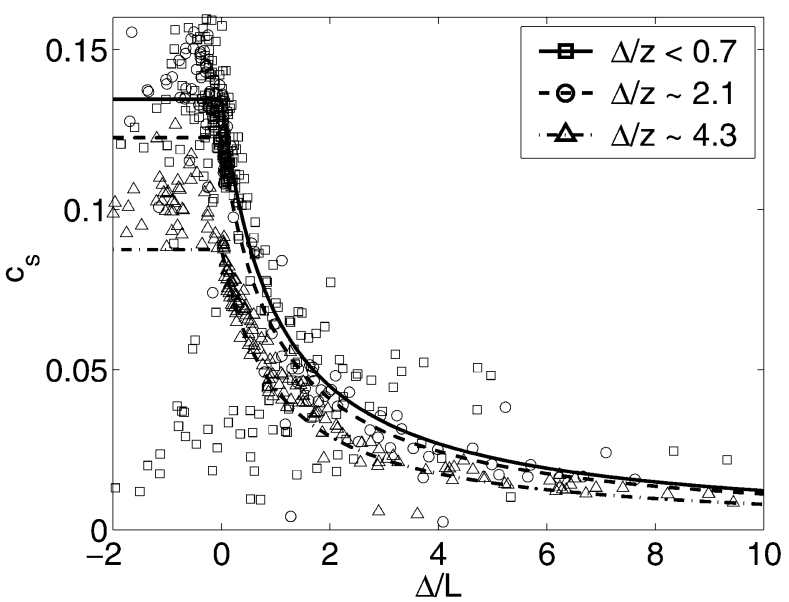

FIG. 4. Smagorinsky coefficient $c_{s}$ as a function of $\Delta / L$ for an averaging time of $T_{c}=13.7 \mathrm{~min} \sim 283 \Delta\langle u\rangle$ for three different values of $\Delta / z$. The symbols represent experimental results, the lines are empirical fits described in Eq. (17).

nificantly for near-neutral conditions, while the pdf in stable regions is almost unchanged. The most likely value for $c_{s}^{2}$ is very similar to Fig. 3a. Moreover, there are fewer events of negative $c_{s}^{2}$.

The mean and the variability of $c_{s}^{2}$ around the most likely, or average, value and the statistics of backscatter events will be addressed in more detail in section 5 . Next, we include the effects of distance to the ground (by considering results from different arrays).

Figure 4 shows results for $c_{s}$ from averaging over segments of length $T_{c}=T_{L}=13.7 \mathrm{~min} \sim 283 \Delta /\langle u\rangle$ for the four different arrays. The data for arrays 3 and 4 are combined since they correspond to similar values of $\Delta / z$. As is visible, even after averaging over times corresponding to 283 filter length scales, there is significant variability. Nevertheless, it is seen that for all stabilities, the $c_{s}$ values for large $\Delta / z$ tend to fall below those for low $\Delta / z$, a trend that is consistent with previous results (Mason 1994; Porté-Agel et al. 2000a, 2001b). In order to identify more clearly the trends with $\Delta / L$ and $\Delta / z$, averages are performed over the entire data available.

Figure 5 shows results for $c_{s}$ from averaging SGS energy dissipations over all segments within each $\Delta / L$ bin of Fig. 2. Thus, these results correspond to using $T_{c}$ equal to the times indicated in Fig. 2 in each case. A very clear dependence of the coefficient on $\Delta / L$ and $\Delta / z$ can be identified. Considering the heterogeneity of the data within one bin with respect to wind angle, turbulence intensity, mean velocity, etc., it is reassuring that such clear trends emerge from the data. From its neutral value, $c_{s}$ decreases strongly under stable atmospheric conditions. Moreover, a larger $\Delta / z$ leads to a decrease in the model coefficient, consistent with the use of damping functions for $c_{s}$ close to the wall, where $z$ becomes equal to or smaller than $\Delta$.

Based on the data in Fig. 5, a functional dependence 


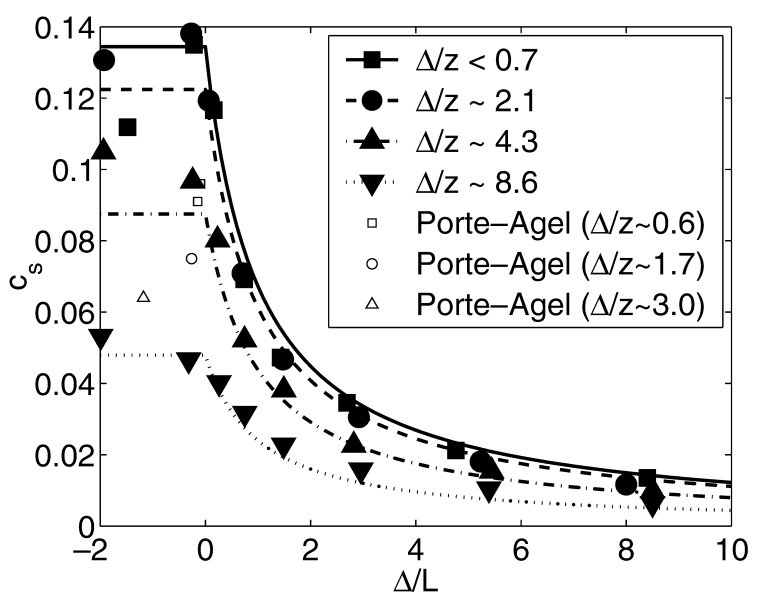

FIG. 5. Smagorinsky coefficient $c_{s}$ as a function of $\Delta / L$ and $\Delta / z$. Data segments of length $T_{L}=6.8 \mathrm{~min}$ are classified according to their $\Delta / L$ values, for each of the four arrays. For each $\Delta / L$ value, Eq. (7) is applied to obtain $c_{s}$ using time averages of nominator and denominator over all segments. Depending on the availability of data in each $\Delta / L$ bin, the averaging time ranges from $T_{c}=0.8 \mathrm{~h}$ to $T_{c}=$ $22.9 \mathrm{~h}$. The symbols represent these experimental results, the lines are empirical fits described in Eq. (17). To test the fit for a different $\Delta / z$ value, $c_{\text {s }}$ is recomputed for a larger filter size $\Delta / z \sim 8.6$ using data from array 1 (downward facing triangles). Results obtained by Porté-Agel et al. (2001b) are included as open symbols.

of $c_{s}$ on both $\Delta / L$ and $\Delta / z$ is constructed. To establish a functional dependence of $c_{s}$ on $\Delta / z$, Eq. (6) for nearneutral stratification is written as

$$
c_{s}=c_{0}\left[1+\left(\frac{c_{0}}{\kappa} \frac{\Delta}{z}\right)^{n}\right]^{-1 / n} .
$$

In addition, for stable stratification $c_{s}$ has to be decreased compared to its value in neutral conditions. Considering the trends shown in Fig. 6a (which corresponds to Fig. 5 for $\Delta / L>0$ but plotted in $\log -\log$ coordinates to identify possible power-law scaling) we conclude that $c_{s}$ decreases as $c_{s} \sim(\Delta / L)^{-1}$ in very stable conditions for fixed $\Delta / z$. In other words, the length scale $l=c_{s} \Delta$ scales as $L$ in stably stratified conditions. This is consistent with results presented in Sullivan et al. (2003, hereafter SUL) who show that $l$ scales with the peak in the spectrum of vertical velocity. That length scale is known to scale with $L$ (Nieuwstadt 1984).

Thus, a correction factor appropriate for the stable range is $\left(1+\left(c_{0} / \alpha\right)(\Delta / L)\right)^{-1}$, where $\alpha=O\left(c_{0}\right)$ is a model parameter. For large $\Delta / L$ this converges to $\left(\alpha / c_{0}\right)$ $(\Delta / L)^{-1}$, whereas for small (but positive) $\Delta / L$ it approaches 1. Combining this expression with Eq. (16), and introducing the Ramp function $R(x)[R(x)=x$ if $x$ $>0$ and $R(x)=0$ if $x<0$ ] to avoid difficulties in the unstable range where $L<0$, we propose an expression of the form

$$
c_{s}=c_{0}\left[1+\frac{c_{0}}{\alpha} R\left(\frac{\Delta}{L}\right)\right]^{-1}\left[1+\left(\frac{c_{0}}{\kappa} \frac{\Delta}{z}\right)^{n}\right]^{-1 / n} .
$$
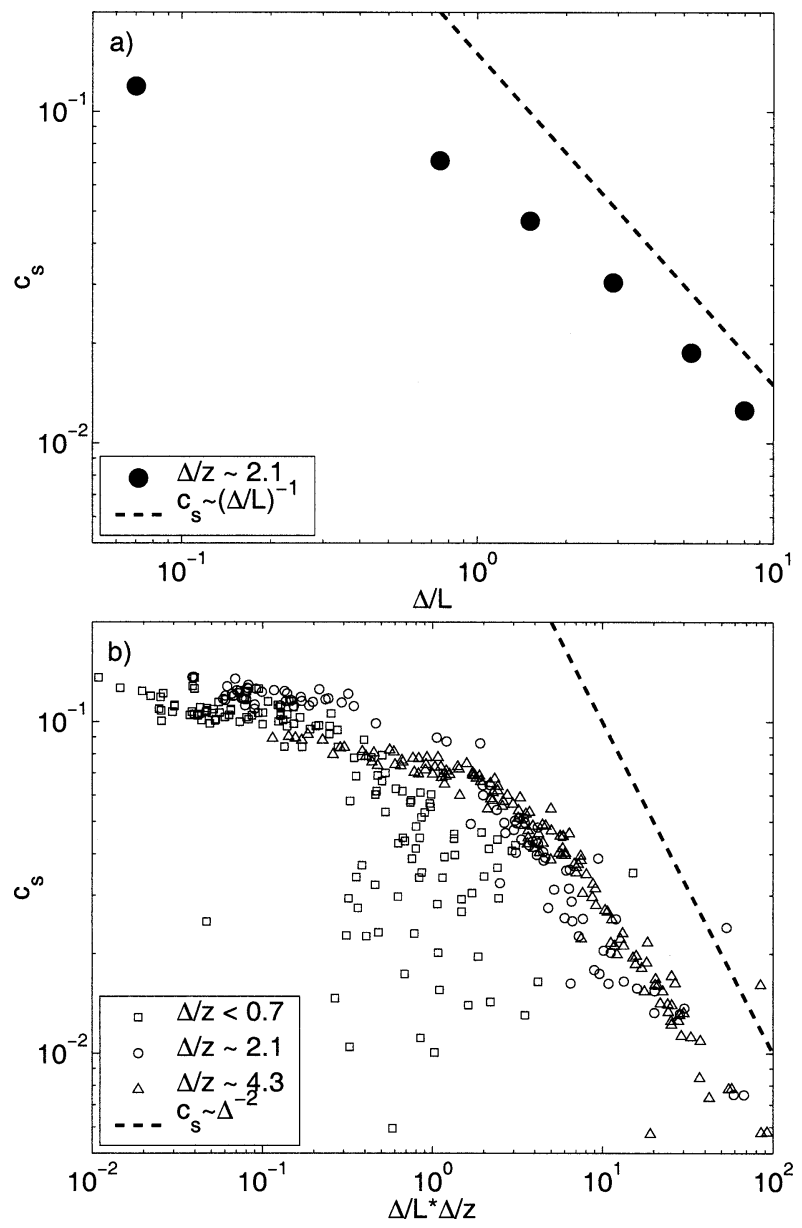

FIG. 6. (a) Same as Fig. 5 for $\Delta / L>0$, but plotted in log-log coordinates to identify possible power-law scaling. The dashed line shows a $(\Delta / L)^{-1}$ scaling. (b) Smagorinsky coefficient $c_{s}$ as a function of $\Delta / L \times \Delta / z$ for an averaging time of $T_{c}=13.7 \mathrm{~min} \sim 283 \Delta\langle u\rangle$. The symbols represent experimental results; the dashed line shows a $c_{s} \sim\left(\Delta^{2}\right)^{-1}$ scaling.

To further examine the validity of the proposed expression we consider the simultaneous limit of large $\Delta / L$ and large $\Delta / z$. For this limit (and $n \geq 1$, say), Eq. (17) reduces to $c_{s} \sim(\Delta / L)^{-1}(\Delta / z)^{-1}$. To test this asymptotic trend, in Fig. $6 \mathrm{~b}, c_{s}$ is plotted versus $\Delta / z \times \Delta / L$ for all arrays. Indeed, for large $\Delta / L$ and large $\Delta / z c_{s}$ follows closely the line $c_{s} \sim\left(\Delta^{2} /(L z)\right)^{-1}$, justifying the proposed fit in Eq. (17). This suggests that for $\Delta \gg L$ and $\Delta \gg$ $z$ the value of $c_{s}$ is determined by the product of the two length scales $L$ and $z$ rather than by the smaller of the two.

To fit the parameters of Eq. (17) to the data in Fig. 5 we set $n=3$ and fit $c_{0}$ and $\alpha$ using multidimensional unconstrained nonlinear optimization from MATLAB. Mason and Brown (1999) suggest $n=2$, but the small differences between the $c_{s}$ of different arrays in neutral and unstable conditions are indication of a slower decrease of $c_{s}$ with $\Delta / z$, which requires a larger n. From the optimization with $n=3$, we obtain $c_{0}=0.1347$, 


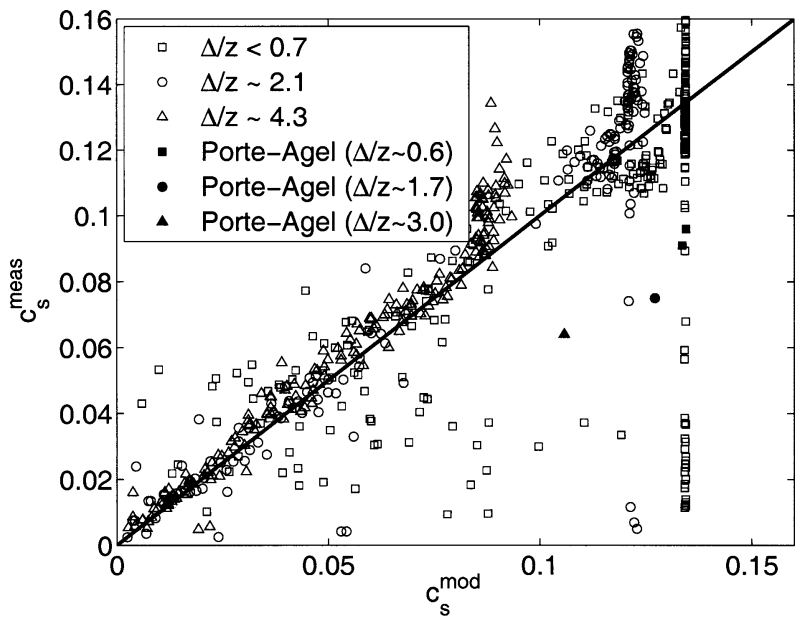

FIG. 7. Scatterplot of measured vs modeled results for the Smagorinsky coefficient $c_{s}$ for an averaging time of $T_{c}=13.7 \mathrm{~min}$. The symbols represent experimental results, the line marks $c_{s}^{\text {meas }}=c_{s}^{\text {mod }}$. The expression used to compute $c_{s}^{\text {mod }}$ is described in Eq. (17). Results obtained by Porté-Agel et al. (2001b) are included as filled symbols.

and $\alpha=0.1289$. Since the difference between $c_{0}$ and $\alpha$ is within the range of experimental uncertainty, we assume $\alpha=c_{0}=0.135$. The resulting equation is used for the fits in Fig. 5, as well as in the preceding Figs. 3 and 4.

The proposed fit is tested by comparison with a different set of data, namely from array 1 in which a box filter is applied on four adjacent sonics in the s array and the corresponding sonics in the $\mathrm{d}$ array. This results in a filter scale of $\Delta=26.8 \mathrm{~m}$ and a value of $\Delta / z=$ 8.6. Using a one-sided derivative in the $y$ direction and a centered derivative in the $x$ direction, the quantities needed to compute $c_{s}^{2}$ from Eq. (7) are obtained and the results are shown in Fig. 5 as downward facing triangles. We conclude that the proposed model fits these test data quite well.

As a further test of the proposed fit, Fig. 7 compares the measured $c_{s}$ for an averaging time $T_{c}=T_{L}=13.7$ min with the value obtained from Eq. (17). It can be concluded that the empirical fit represents the mean trends in the data also for the shorter (compared to Fig. 5) averaging time. However, for unstable conditions (large $c_{s}$ ), deviations between the modeled and the measured $c_{s}$ occur due to the large variability of the measured $c_{s}$, whereas the model fit yields a constant value of $c_{s}$ for any given value of $\Delta / z$. Also, for arrays 3 and $4(\Delta / z<0.7)$ the scatter in the data is larger than for arrays 1 and 2 . This might be caused by the difference in setup geometry of array 3 . There, the single-filtered array is below the double-filtered array (see Table 1), which influences and possibly overestimates vertical derivatives compared to the other setups. For array 4, different filter types in the lateral direction are used for the single- and double-filtered arrays, as indicated in Table 1.

Analyses by other investigators have revealed similar

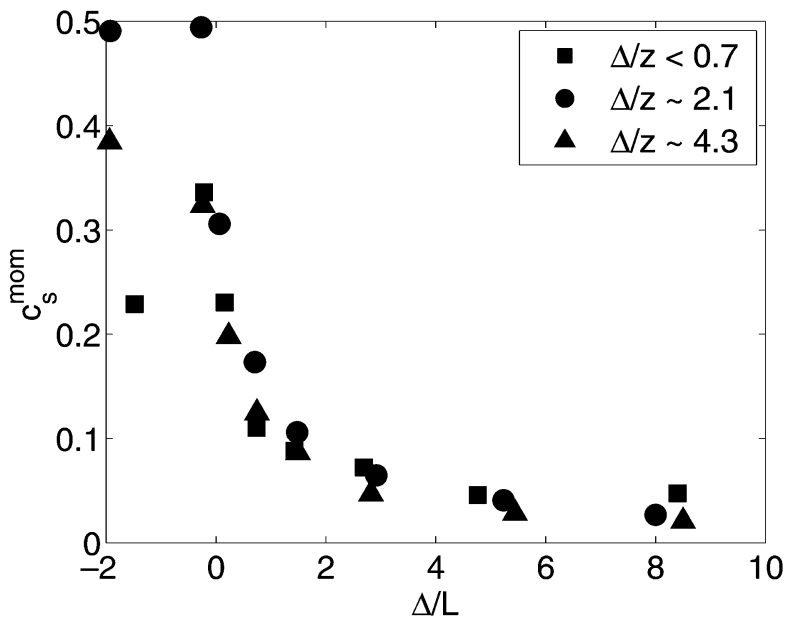

FIG. 8. Smagorinsky coefficient $c_{s}^{\text {mom }}=\left[-\left\langle\tau_{13}\right\rangle /\left\langle 2 \Delta^{2}|\tilde{S}| \tilde{S}_{13}\right\rangle\right]^{1 / 2}$ as a function of $\Delta / L$ and $\Delta / z$. Averages are evaluated over the entire dataset.

results. Deardorff (1971) and Piomelli et al. (1988) both found $c_{s} \approx 0.1$ for small $\Delta / z$. Porté-Agel et al. (2001a) found $c_{s} \approx 0.08$ which is about $35 \%$ smaller than ours, but the tendency of an increase of the coefficient with $\Delta / z$ is the same.

The proposed expression in Eq. (17) can be easily used in LES, since $\Delta / L$ and $\Delta / z$ are known parameters that are imposed in the simulations a priori by the choice of mesh spacing, wall shear stress, and heat flux at the boundary. If the dependence on stratification is to be expressed as function of Richardson number, relationships between $\mathrm{Ri}$ and $L / z$ can be used such as those appearing in Businger et al. (1971). However, most of the recent work dealing with stability of the lower atmosphere has tended to be in terms of $L$ (Brutsaert 1982).

Finally, we report the coefficient values that are obtained from matching momentum flux instead of dissipation, according to Eq. (13). Figure 8 shows the coefficients so determined for various $\Delta / z$ and $\Delta / L$. Comparing with Fig. 5, we see that the coefficients are much larger. LES with such values are known to be overly damped and thus we conclude that the condition of correct energy dissipation is more appropriate for the data analysis. The impossibility to choose a $c_{s}$ that satisfies both the requirements of producing the correct rate of kinetic energy transfer from the resolved to the subgridscales $\Pi$ and the correct subgrid-scale stress $\tau_{i j}$ is a basic flaw of the eddy-viscosity model. For further information consult Meneveau (1994), Pope (2000, p. 603), and Juneja and Brasseur (1999).

\section{Dependence of $c_{s}$ on local strain-rate magnitude}

The basic scaling inherent in the Smagorinsky model, predicated upon inertial-range dimensional arguments, assumes that the eddy viscosity is linearly proportional 


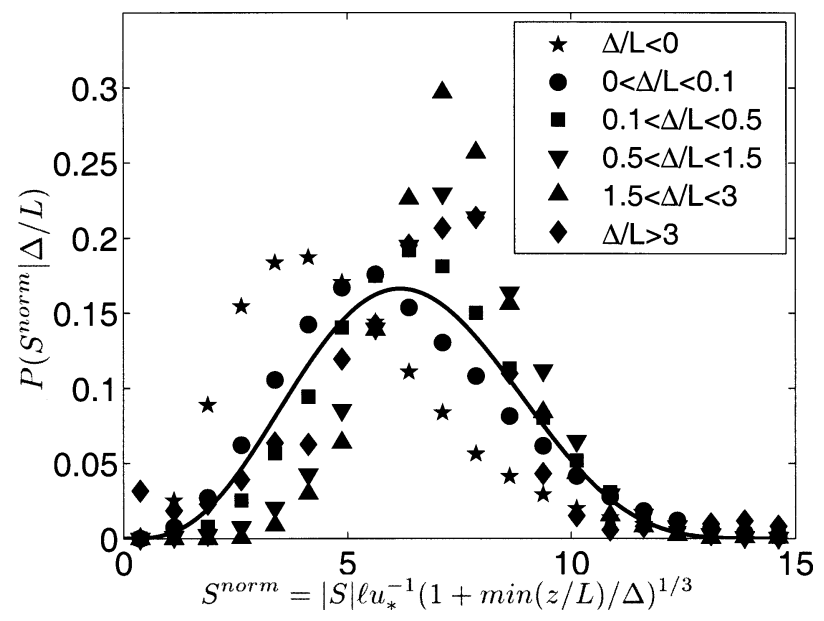

FIG. 9. Probability density function of strain-rate magnitude normalized by $u_{\Delta} / \ell$ conditioned on $\Delta / L$. The scales used to normalize $|\tilde{S}|$ are $u_{\Delta}=u_{*}(1+\min (z, L) / \Delta)^{-1 / 3}$ as velocity scale and the empirical fit of Eq. (18) as length scale $\ell$. The data are from array $1(\Delta /$ $z \sim 4.3$ ). For clarity, a smooth beta distribution is fit to the (unconditioned) pdf of $S^{\text {norm }}$ (solid line).

to the local strain-rate magnitude $|\tilde{S}|$ [see Eq. (3)]. Whether this concept is justified can be examined by evaluating $c_{s}$ from subsets of the data in which $|\tilde{S}|$ has certain values. If the Smagorinsky scaling is correct, the measured value of $c_{s}$ should be independent of strainrate magnitude. Thus, in this section we further classify the available data according to the local strain-rate magnitudes for conditional sampling. Since the data must also be classified into different ranges of stabilities, the limited amounts of data under each condition become an issue. In order to assure sufficient amounts of data in each condition, data segments of $T_{L}=6.8 \mathrm{~min}$ are classified into six ranges of stability - unstable to neutral $(\Delta / L \leq 0)$ - and several ranges of increasing stability$(0<\Delta / L<0.1,0.1<\Delta / L<0.5,0.5<\Delta / L<1.5$, $1.5<\Delta / L<3$, and $\Delta / L>3$ ).

For each of the stability ranges, we consider the pdf of the filtered strain-rate magnitude $|\tilde{S}|$ to decide how many bins of $|\tilde{S}|$ to use for conditional sampling. As expected, the pdfs and ranges of variability of $|\tilde{S}|$ depend on stability. We seek to collapse the range of pdfs by normalizing the strain-rate magnitude by a velocity scale $u_{\Delta}$ and a length scale $\ell$ appropriate to the values of $\Delta / L$ and $\Delta / z$. For consistency with the empirical fits of section 3 , we use the length scale

$$
\ell=\Delta\left[1+R\left(\frac{\Delta}{L}\right)\right]^{-1}\left[1+\left(\frac{c_{0}}{\kappa} \frac{\Delta}{z}\right)^{n}\right]^{-1 / n} .
$$

Only when $\Delta \ll z$ and $\Delta \ll L$, one obtains the standard filter scale $\ell \sim \Delta$. As velocity scale, we use the inertial-range scaling $u_{\Delta} \sim u_{*}(\Delta / z)^{1 / 3}$ when $\Delta<\min (z$, $L)$. Otherwise, when $\Delta>\min (z, L), u_{*}$ is a reasonable velocity scale. The velocity scale $u_{\Delta}=u_{*}[1+\min (z$, $L) / \Delta]^{-1 / 3}$ combines these two scaling behaviors. The normalized strain rate is then defined as

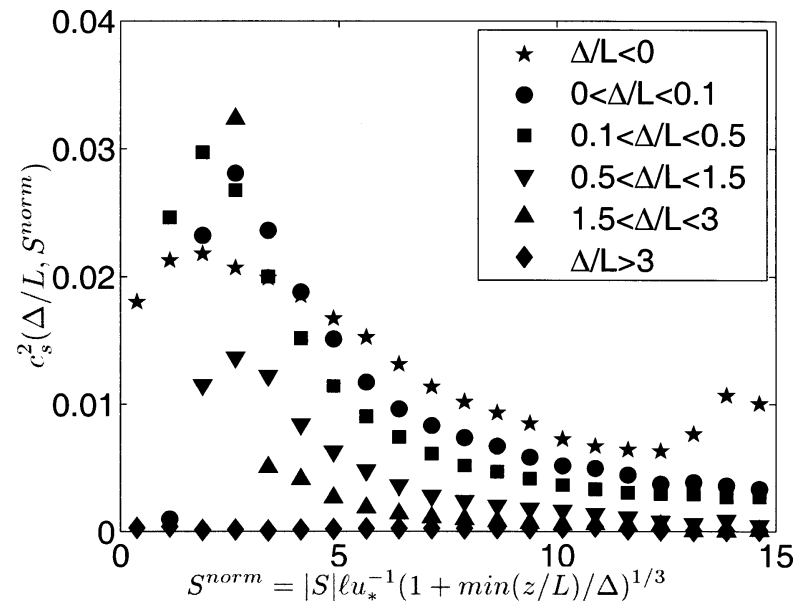

FIG. 10. Smagorinsky coefficient $c_{s}^{2}$ conditioned on normalized strain-rate magnitude $S^{\text {norm }}=|\tilde{S}| \ell / u_{\Delta}$ for different $\Delta / L$. The scales used to normalize $|\tilde{S}|$ are $u_{\Delta}=u_{*}(1+\min (z, L) / \Delta)^{-1 / 3}$ as a velocity scale and the empirical fit of Eq. (18) as a length scale $\ell$. The data are from array $1(\Delta / z \sim 4.3)$.

$$
S^{\text {norm }} \equiv|\tilde{S}| \frac{\ell}{u_{*}}\left[1+\frac{\min (z, L)}{\Delta}\right]^{1 / 3},
$$

with $\ell$ given by Eq. (18). Figure 9 shows the pdfs of $S^{\text {norm }}$ for the various $\Delta / L$ cases for array 1 . It can be observed that the range of $S^{\text {norm }}$ is roughly independent of $\Delta / L$ with most of the data falling between $S^{\text {norm }}=1$ and $S^{\text {norm }}=10$, although the collapse of the different pdfs is not very good. The magnitude of the normalized strain-rate is smaller in unstable conditions and gradually increases in slightly stable conditions. In very stable conditions the pdfs look similar for different ranges of $\Delta / L$. Of the many different normalizations of $|\tilde{S}|$ we have attempted, Eq. (19) and Fig. 9 show the least scattered results and we conclude that our normalization is appropriate for present purposes.

The range $0<S^{\text {norm }}<15$ is divided into 20 strainrate bins, and the conditional Smagorinsky coefficient is computed from the data. The coefficient is evaluated as follows:

$$
c_{s}^{2}\left(\frac{\Delta}{L}, S^{\text {norm }}\right)=-\frac{\left\langle\tau_{i j} \tilde{S}_{i, j} \mid \frac{\Delta}{L}, S^{\text {norm }}\right\rangle}{\left\langle 2 \Delta^{2}|\tilde{S}| \tilde{S}_{i j} \tilde{S}_{i j} \mid \frac{\Delta}{L}, S^{\text {norm }}\right\rangle} .
$$

The conditional averages are evaluated over the entire set of available data points within each bin. Figure 10 shows $c_{s}^{2}$ as function of normalized strain-rate for each of the stability ranges considered, for the case $\Delta / z \sim$ 4.3 (array 1). As already shown in section 3, $c_{s}$ decreases with increasing stability. The observed trends with strain-rate magnitude are as follows. For unstable conditions $(\Delta / L<0), c_{s}^{2}$ decreases with strain-rate magnitude but only by a factor of about 2 - values decrease 

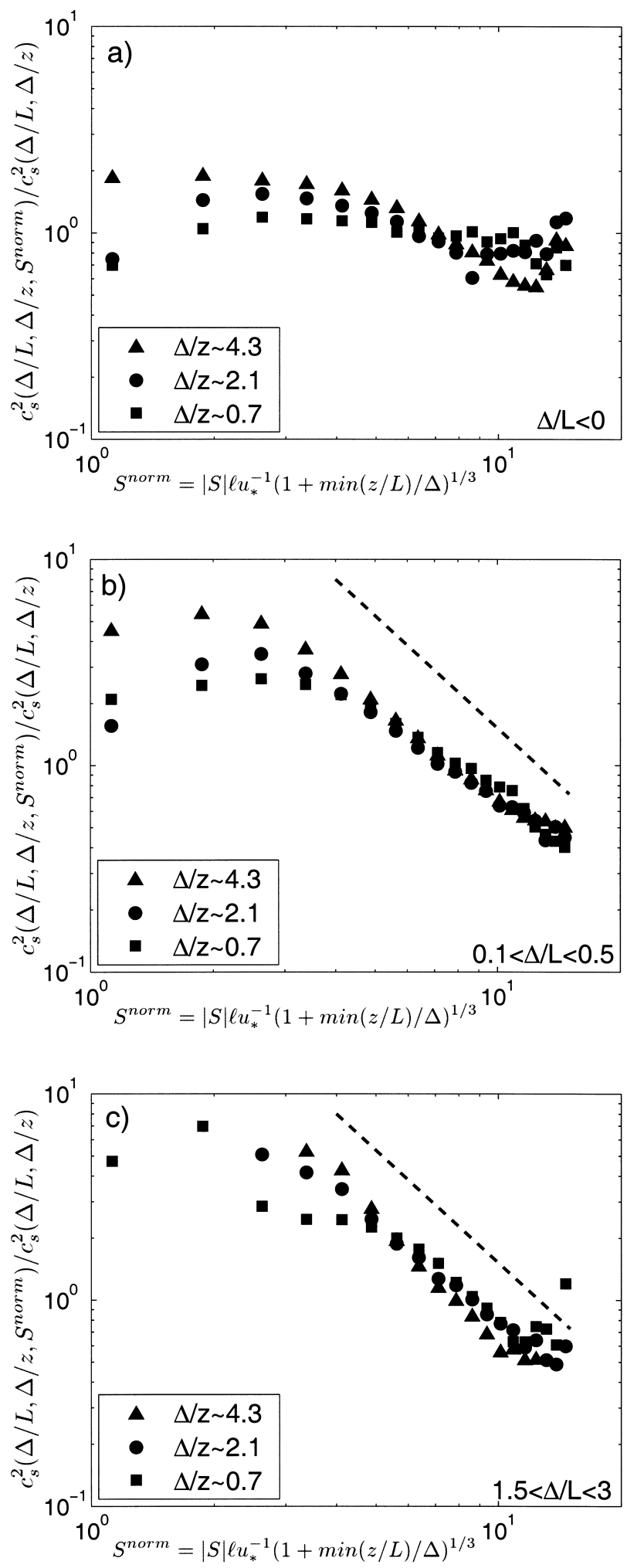

FIG. 11. Log-log plots of Smagorinsky coefficient $c_{s}^{2}$ conditioned on normalized strain-rate magnitude $S^{\text {norm }}$ for different stabilities $\Delta /$ $L$ and arrays. (a) Unstable: $\Delta / L<0$; (b) slightly stable: $0.1<\Delta / L$ $<0.5$; (c) very stable: $1.5<\Delta / L<3$. In (b) and (c), the dashed line has a slope of -1 and shows an inverse power-law behavior. from $c_{s}^{2} \sim 0.02$ at low $S^{\text {norm }}$ to $c_{s}^{2} \sim 0.01$ at high $S^{\text {norm }}$. We remark that trends for $S^{\text {norm }}<2$ are rather inconclusive and appear noisy, probably due to the small amount of data available in these bins. In stable stratification (except for the case $\Delta / L>3$, which shows negligibly small coefficients from which no trend with strain-rate can be discerned), the coefficient decreases quite significantly with increasing local strain-rate magnitude. Typically the coefficient decreases about fivefold between $S^{\text {norm }}=2$ and $S^{\text {norm }}=10$.

In order to isolate the effect of strain-rate magnitude, the conditional $c_{s}^{2}$ values are normalized by $c_{s}^{2}(\Delta / L)$, the Smagorinsky coefficient conditioned on $\Delta / L$ for each array obtained by summing over all strain-rate bins. Figure 11 compares these normalized $c_{s}^{2}$ for all three arrays, by considering different stability ranges. Figure $11 \mathrm{a}$ is for unstable cases, Fig. 11b is for slightly stable cases, and Fig. 11c is for very stable cases. For large strain-rate magnitudes, a scaling of $c_{s}^{2} \sim\left(S^{\text {norm }}\right)^{-1}$ can be identified for $1.5<\Delta / L<3$ in Fig. 11c. This slope becomes smaller in magnitude when $\Delta / L$ approaches zero (Fig. 11b) and $c_{s}^{2}$ is found to be almost constant in unstable atmospheric conditions (Fig. 11a). These trends are similar for all $\Delta / z$ values (arrays). In terms of normalized strain-rate magnitude, two regimes are identified. For large strain-rate magnitudes, $c_{s}^{2}$ decreases with $S^{\text {norm }}$. The other regime concerns small strain-rate magnitudes and shows an almost constant Smagorinsky coefficient. The transition between these two regimes occurs at values of $S^{\text {norm }}$ that depend on $\Delta / L$ and $\Delta / z$. The smaller $\Delta / L$ and the smaller $\Delta / z$, the smaller the transition value of $S^{\text {norm }}$. Figure $11 \mathrm{~b}$ exemplifies this statement. For $\Delta / z<0.7$ the transition region starts at $S^{\text {norm }} \sim 4$, for $\Delta / z \sim 2.1$ the value is $S^{\text {norm }} \sim 3$ and for $\Delta / z \sim 4.3$ we find $S^{\text {norm }} \sim 2$.

The implications for the Smagorinsky model are as follows. We conclude that the deeper $\Delta$ is in the inertial range $[\Delta \ll \min (z, L)]$ the more $c_{s}^{2}$ is constant with $S^{\text {norm }}$ implying that the Smagorinsky scaling is valid. This becomes especially clear for the unstable to neutral data for which the weak dependence of $c_{s}^{2}$ upon local strainrate magnitude for all arrays provides support for the basic scaling of the Smagorinsky model. However, the data for the stable cases show that the Smagorinsky scaling is erroneous under conditions of stable stratification. As a consequence, one may conclude that to properly scale the eddy-viscosity one must not only change the basic length scale (i.e., using $\ell$ as opposed to $\Delta$ ) but also the velocity scale. More specifically, results suggest that at large $S^{\text {norm }}$ and $\Delta / L$, the coefficient of Eq. (17) should be multiplied by a factor $[1+\beta(\Delta /$ $\left.L) S^{\text {norm }}\right]^{-1}$ where $\beta(\Delta / L)$ is some function that describes at what $S^{\text {norm }}$ the transition to a $\left(S^{\text {norm }}\right)^{-1}$ occurs. In the limit of large $S^{\text {norm }}$, the eddy viscosity would then scale as $c_{0}^{2} \ell^{2}\left(S^{\text {norm }}\right)^{-1}|\tilde{S}| \sim c_{0}^{2} \ell u_{\Delta}$ with $u_{\Delta}=u_{*}$ (at large $\Delta /$ $L$ ), instead of $c_{0}^{2} \ell^{2}|\tilde{S}|$. The reasonable collapse in our analysis suggests that the velocity scale $u_{\Delta}$ may be more generally appropriate than the conventional choice of 

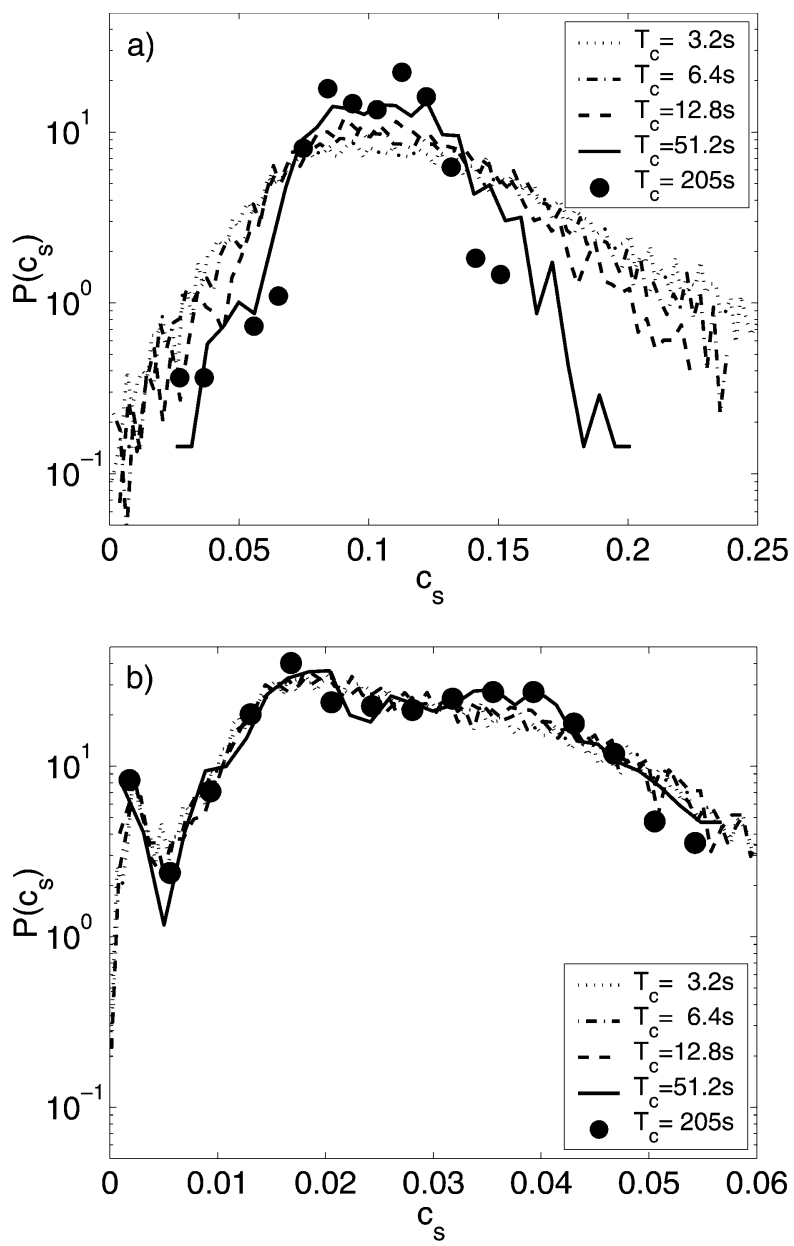

FIG. 12. Pdf of Smagorinsky coefficient $c_{s}$ for different averaging times $T_{c}$ (see legend) for (a) unstable atmospheric stability conditions $(-2.0<\Delta / L<0.0)$ and (b) very stable atmospheric stability conditions $(1.5<\Delta / L<5.5)$. The advection time through one filter scale is roughly $\Delta /\langle u\rangle=5.4 \mathrm{~s}$. The data are from array $1(\Delta / z \sim$ 4.3).

$\ell|\tilde{S}|$. Finally, we recall that one has to differentiate between the scaling with local strain rate as it is examined here and the dependence on global shear as examined in Hunt et al. (1988) and Canuto and Cheng (1997). In this paper we consider the dependence on global shear in the case of near-wall ABL to be already subsumed by the dependence upon $\Delta / z$ that was considered in section 3 .

\section{Variability of $c_{s}$}

In this section we address the question "how variable is $c_{s}$ ?" Results shown in section 3, specifically Figs. 3a and $3 \mathrm{~b}$, suggest that while the most likely value of $c_{s}^{2}$ does not change significantly with averaging time $T_{c}$, the variability of the coefficient decreases for increasing $T_{c}$, at least for the near-neutral and unstable cases. To quantify the dependence of the statistics of $c_{s}$ on $T_{c}$ and stability, pdfs of $c_{s}$ are computed for different values of
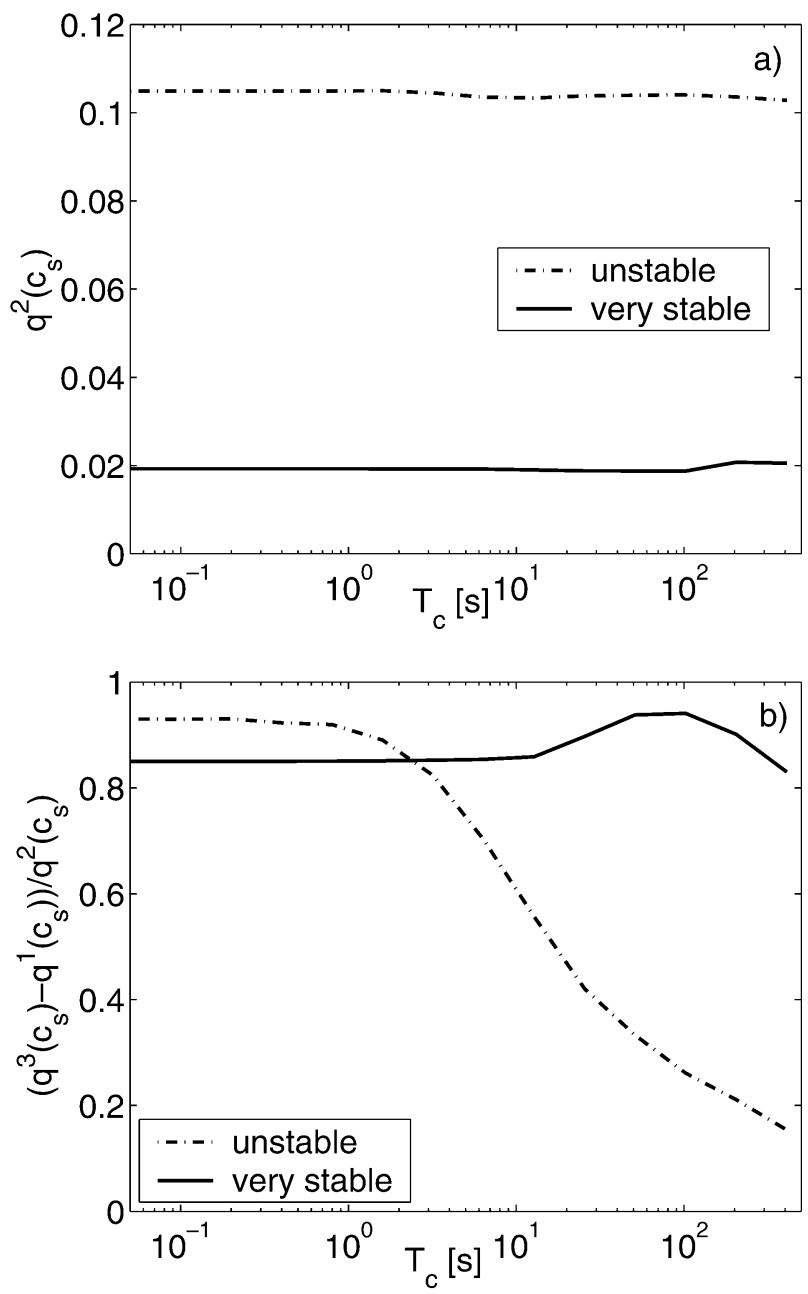

FIG. 13. (a) Median of pdf of Smagorinsky coefficient $c_{s}$ and (b) width of pdf of $c_{s}$ quantified as $\left[q^{3}\left(c_{s}\right)-q^{1}\left(c_{s}\right)\right] / q^{2}\left(c_{s}\right)\left(q^{i}\right.$ means $i$ th quartile) as a function of averaging time $T_{c}$. To contrast unstable and very stable conditions, two stability bins for unstable $(-2.0<\Delta / L$ $<0.0)$ and very stable $(1.5<\Delta / L<5.5)$ atmospheric conditions are selected. The data are from array $1(\Delta / z \sim 4.3)$.

$\Delta / L$ and $T_{c}$. Two stability bins are selected for the analysis. The first bin contains unstable atmospheric conditions characterized by $-2.0<\Delta / L<0.0$. The second bin groups data under very stable conditions. Since there are less overall data available for large $\Delta / L$, in order to obtain reasonably well-converged pdfs, we choose a wide bin of stabilities, namely $1.5<\Delta / L<5.5$. Five different values of $T_{c}$ are selected, ranging from $T_{c}=$ $3.2 \mathrm{~s}$ to $T_{c}=205 \mathrm{~s}$. Figure 12a shows the resulting pdfs for the unstable data, while the very stable data are presented in Fig. 12b. Backscatter events are excluded from the analysis to focus on $c_{s}>0$. The probability $P\left(c_{s}^{2}<0\right)$ is less than 0.2 (see Fig. 14).

Figure 12a shows that the spread in the pdf of $c_{s}$ increases for decreasing $T_{c}$ for unstable atmospheric stability. Reassuringly, however, the most likely value of $c_{s}$ and the median (as shown in Fig. 13a) do not depend 


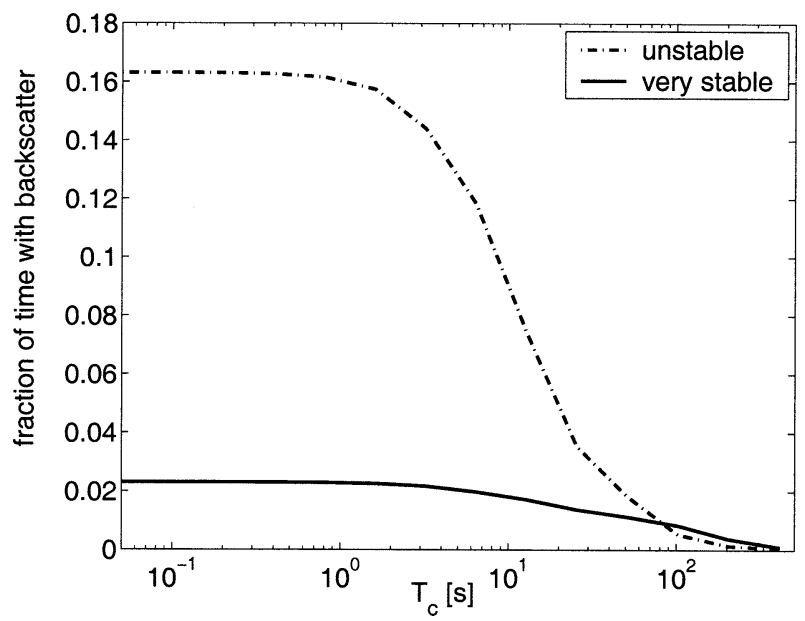

FIG. 14. Fraction of segments of length $T_{c}$ with negative $c_{s}^{2}$ as a function of averaging time $T_{c}$ for unstable $(-2.0<\Delta / L<0.0)$ and very stable $(1.5<\Delta / L<5.5)$ conditions. The data are from array 1 .

on $T_{c}$. For stable conditions (Fig. 12b), the most likely value and the median (Fig. 13a) of $c_{s}$ are constant with $T_{c}$ and smaller than for unstable conditions, in agreement with the findings in section 3 . The fact that the medians of $c_{s}$ are independent of $T_{c}$ for stable and unstable conditions is encouraging for LES with dynamic SGS models which, as discussed in the introduction, often use some kind of averaging procedures, either in space (e.g., horizontal planes) or time [e.g., the Lagrangian dynamic model (Meneveau et al. 1996)] to compute the coefficient. Our results suggest that correct median coefficients can be obtained even for fairly short averaging timescales. Rather surprisingly, however, in the case of stable conditions it appears that the spread in the pdf does not decrease for increasing $T_{c}$.

Figure $13 \mathrm{~b}$ presents a quantification of the width of the pdfs as function of $T_{c}$. Instead of computing the rms value (which tends to be biased due to some outliers in the distribution), we quantify the spread of the pdfs with quartiles. The figure shows the difference between the third and first quartile of the distribution, normalized by the second quartile (thus giving a dimensionless measure of the variability that is not strongly affected by atypical outliers). The relative width of the pdf for the stable bin does not decrease as $T_{c}$ is increased. This result shows strong variability of the real and/or modeled SGS dissipation under stable atmospheric conditions indicating that fluctuations occur over very long timescales. This may be related to the strong intermittency in stable atmospheric conditions.

The fraction of segments of length $T_{c}$ that display average backscatter (with negative $c_{s}^{2}$ over the time $T_{c}$ ) that were neglected in the preceding analysis of section 5 is shown in Fig. 14 as function of $T_{c}$. As expected, the fraction diminishes with increasing $T_{c}$ because backscatter events tend to be cancelled by forward-scatter events within the time-interval $T_{c}$, yielding a positive
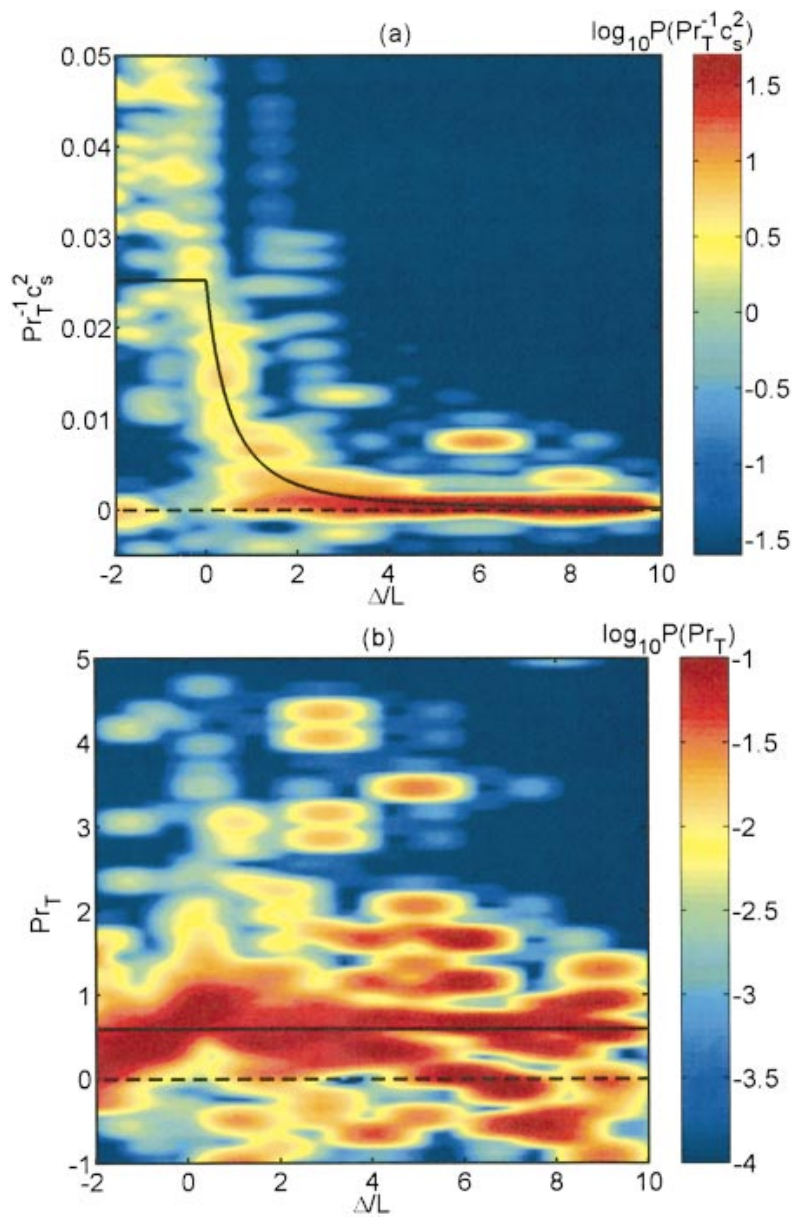

FIG. 15. Contour plot of conditional pdf of (a) $\operatorname{Pr}_{T}^{-1} c_{s}^{2}: P\left(\operatorname{Pr}_{T}^{-1} c_{s}^{2} \mid \Delta /\right.$ $L)$ and (b) $\operatorname{Pr}_{T}: P\left(\operatorname{Pr}_{T} \mid \Delta / L\right)$. The contours are spaced logarithmically. The averaging time is $T_{c}=102.4 \mathrm{~s}=32 \Delta /\langle u\rangle$ and the plots show data from array 2 with $\Delta / z \sim 2.1$. The dashed lines show $\operatorname{Pr}_{T}^{-1} c_{s}^{2}=$ 0 and $\operatorname{Pr}_{T}=0$, respectively. In (b) the solid line depicts $\operatorname{Pr}_{T}(z / \Delta \sim$ 2.1) $=0.60$ (from Table 2); in (a) the solid line shows $\operatorname{Pr}_{T}^{-1} c_{s}^{2}$, with $c_{s}^{2}$ taken from the empirical fit described in Eq. (17) and $\operatorname{Pr}_{T}=0.60$.

$c_{s}^{2}$ on average. Consistent with SUL we find that the fraction of time with backscatter events increases with $z / \Delta$ (not shown). SUL report a ratio of backscattered energy to total transferred energy of 0.2 for this array configuration.

\section{Results for coefficients in scalar models}

As introduced in Eq. (12), the coefficient for the Smagorinsky model for the SGS heat flux $\operatorname{Pr}_{T}^{-1} c_{s}^{2}$ can be computed by matching SGS dissipations of scalar variance. Similar to Fig. 3b, in Fig. 15a, the conditional pdf of $\operatorname{Pr}_{T}^{-1} c_{s}^{2}$ is presented. The data are from array 2 with averaging time $T_{c}=102.4 \mathrm{~s}$. Similar to Fig. 3b, the coefficient decreases under stable conditions, and shows more variability in unstable conditions.

By dividing $c_{s}^{2}$ by $\operatorname{Pr}_{T}^{-1} c_{s}^{2}$ for each data segment, the Prandtl number is obtained and plotted in Fig. 15b. Most 

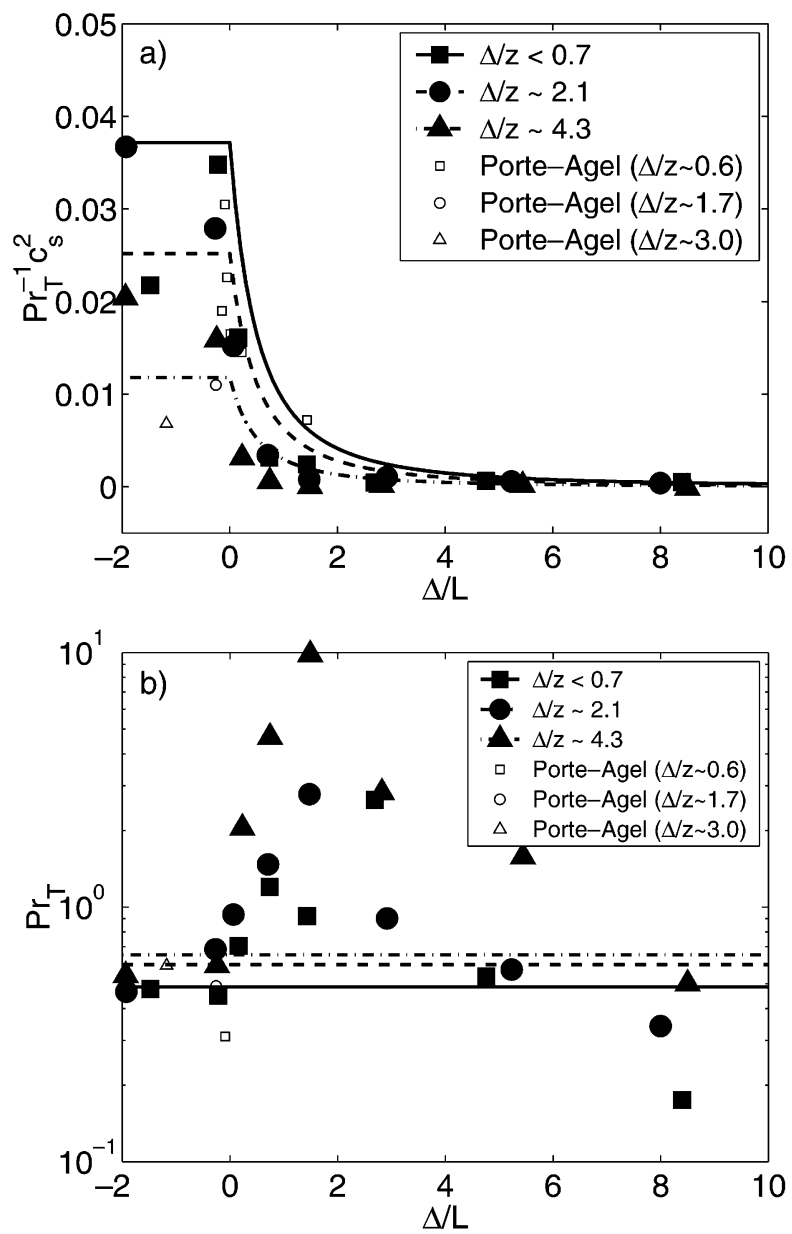

FIG. 16. Smagorinsky model coefficients (a) $\operatorname{Pr}_{T}^{-1} C_{s}^{2}$ and (b) $\operatorname{Pr}_{T}$ as a function of $\Delta / L$ for different $\Delta / z . T_{L}=6.8 \mathrm{~min}$ data segments are classified according to their $\Delta / L$ values, for each of the four arrays. For each $\Delta / L$ value, Eq. (12) is applied to obtain $\operatorname{Pr}_{T}^{-1} c_{s}^{2}$ using time averages of nominator and denominator over all segments. Coefficient $\operatorname{Pr}_{T}$ is computed by dividing $c_{s}^{2}$ computed from Eq. (7) by $\operatorname{Pr}_{T}^{-1} c_{s}^{2}$. Depending on the availability of data in each $\Delta / L$ bin, the averaging time ranges from $T_{c}=0.8 \mathrm{~h}$ to $T_{c}=22.9 \mathrm{~h}$. The lines are empirical fits. The fits are constructed from Eq. (17) (for $c_{s}^{2}$ ) and from Table 2 for $\operatorname{Pr}_{T}$. Results obtained by Porté-Agel et al. (2001b) are included as open symbols.

values of $\operatorname{Pr}_{T}$ lie between 0 and 1 independent of stability. For unstable to neutral conditions, the most likely value of $\operatorname{Pr}_{T}$ increases from $\operatorname{Pr}_{T} \sim 0.3$ to $\operatorname{Pr}_{T} \sim 0.8$, and over the stable range a clear tendency is not apparent. The spread in the conditional pdf does not change significantly with stability. In the following, the dependencies of $\operatorname{Pr}_{T}^{-1} c_{s}^{2}$ and $\operatorname{Pr}_{T}$ are examined in more detail.

Repeating the analysis of section $3, \operatorname{Pr}_{T}^{-1} c_{s}^{2}$ and $\operatorname{Pr}_{T}$ are computed by averaging over the total available time for a given $\Delta / L$ and $\Delta / z$-bin. Figure 16a supports the previous finding that $\operatorname{Pr}_{T}^{-1} C_{s}^{2}$ decreases in stable conditions. For different $\Delta / z$, we observe that $\operatorname{Pr}_{T}^{-1} c_{s}^{2}$ is smaller for $\Delta / z \sim 4.3$ than for $\Delta / z \sim 2.1$ and $\Delta / z<0.7$. For $\mathrm{Pr}_{T}$, the results presented in Fig. 16b are significantly more noisy. Due to the large spread of values for $\operatorname{Pr}_{T}$,
TABle 2. Prandtl number $\operatorname{Pr}_{T}$ conditioned on $\Delta / z$ computed from Eqs. (7) and (12), assuming that $\operatorname{Pr}_{T}$ is not a function of stability. The averaging time is the total time available for each array $\left(T_{c}>\right.$ $35 \mathrm{~h})$.

\begin{tabular}{cccc}
\hline \hline & $\Delta / z \sim 4.3$ & $\Delta / z \sim 2.1$ & $\Delta / z<0.7$ \\
\hline $\operatorname{Pr}_{T}$ & 0.67 & 0.60 & 0.49 \\
\hline
\end{tabular}

the $y$ axis is plotted in logarithmic units. No clear trend of variation with $\Delta / L$ can be discerned from the data, although for this very long averaging time there is a rise in $\operatorname{Pr}_{T}$ between $\Delta / L \sim 1$ and $\Delta / L \sim 4$. However, this trend depends strongly on $T_{c}$. In almost all $\Delta / L$ bins the Prandtl number increases with increasing $\Delta / z$. In order to get a robust estimate on the value of $\operatorname{Pr}_{T}$ for different $\Delta / z$, the Prandtl number is computed by averaging over all stabilities. The results are shown in Table 2 and plotted in Figs. $15 \mathrm{~b}$ and $16 \mathrm{~b}$ as horizontal lines. Indeed, $\operatorname{Pr}_{T}$ is increasing with $\Delta / z$.

In order to quantify the variability of $\operatorname{Pr}_{T}$, the analysis of section 5 is repeated. All data segments with -2.0 $<\Delta / L<0.0$ (unstable bin) and $1.5<\Delta / L>5.5$ (stable bin) are selected and $\operatorname{Pr}_{T}(\Delta / L)$ is computed with varying averaging times $T_{c}$. Then the quartiles of the resulting probability distribution of $\operatorname{Pr}_{T}$ are obtained and the median $q^{2}$ is plotted in Fig. 17a. In contrast to our findings concerning $c_{s}$, the median of the Prandtl number is not constant, but increases with $T_{c}$. This explains the difference between Figs. 16b and 15b, in which $\operatorname{Pr}_{T}$ computed from averages over several hours in Fig. 16b was significantly larger than $\operatorname{Pr}_{T}$ computed from 102.4-s averages in Fig. 15b. The increase with averaging time appears to level off for $T_{c}>10^{2} \mathrm{~s}$. For all $T_{c}$, the median for very stable conditions is larger than the median for unstable conditions, but they seem to converge for large $T_{c}$. A similar behavior (but with different magnitudes of Prandtl numbers) is observed for the other arrays. The dependence of the median of $\operatorname{Pr}_{T}$ on the averaging time and the large scatter in Fig. 15b complicate the development of empirical expressions for $\operatorname{Pr}_{T}$ and $\operatorname{Pr}_{T}^{-1} c_{s}^{2}$. Thus, we only present definitive results on the dependence of $\operatorname{Pr}_{T}$ upon $\Delta / z$ (as shown in Table 2), and refrain from attempting to fit the $\Delta / L$ dependence.

In comparing with prior results, we can remark that for small $\Delta / z$, Mason and Derbyshire (1990), Moin et al. (1991), and Porté-Agel et al. (2001a) found $\operatorname{Pr}_{T} \sim$ 0.4 , which is within the range of uncertainity around our value of $\operatorname{Pr}_{T}(\Delta / z<0.7)=0.49$. For large $\Delta / z$, PortéAgel et al. (2001a) examined two 30-min segments whose $\Delta / z$ roughly correspond to the values for our arrays 1 and 2 . For the setup similar to our array 2 they obtain $\operatorname{Pr}_{T} \sim 0.5$ for $\Delta / L=-0.26$, their analysis of the setup similar to our array 1 results in $\operatorname{Pr}_{T} \sim 0.6$ for $\Delta / L=-1.18$. Our results from Table 2 suggest $\operatorname{Pr}_{T}$ $=0.60$ and $\operatorname{Pr}_{T}=0.67$, which is qualitatively consistent and within the range of experimental uncertainty.

The spread of the pdf of $\operatorname{Pr}_{T}$ is shown in Fig. 17b as a function of $T_{c}$. For unstable atmospheric stability con- 

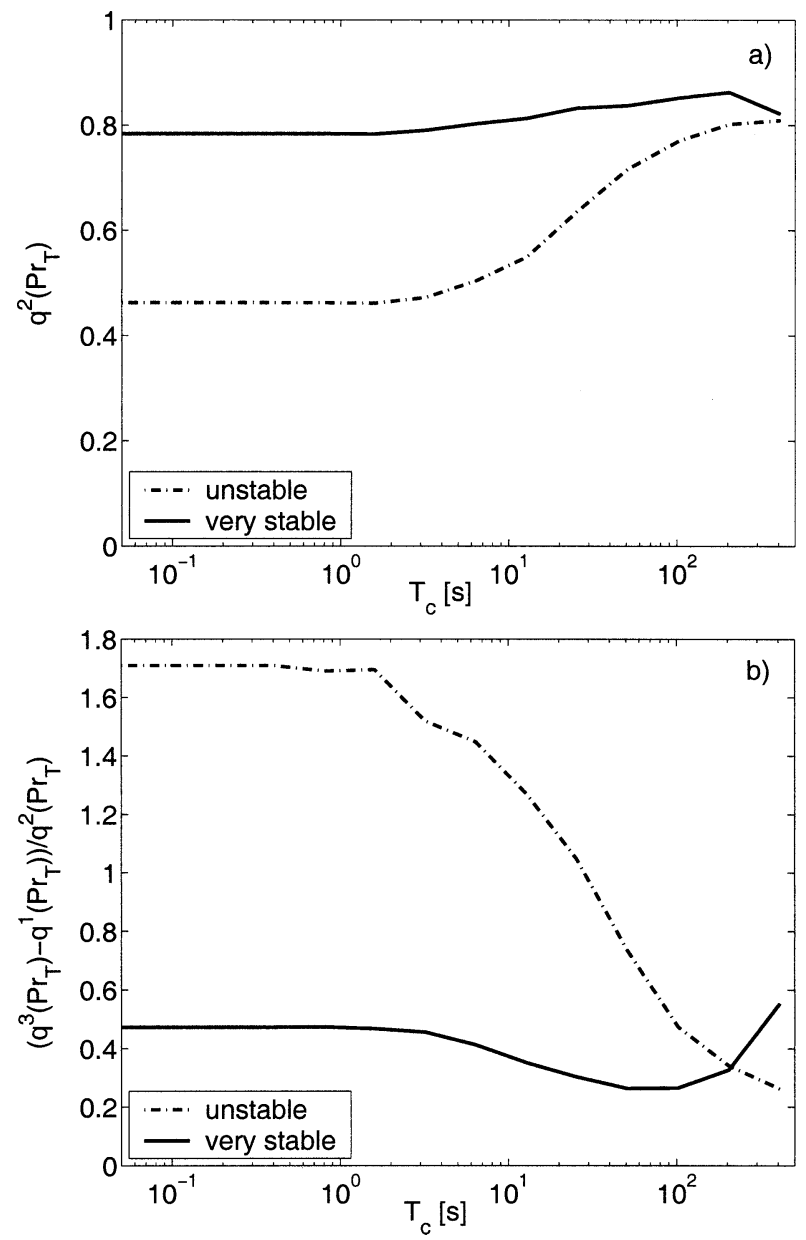

FIG. 17. (a) Median of pdf of Prandtl number $q^{2}\left(\operatorname{Pr}_{T}\right)$ and (b) width of pdf of $\operatorname{Pr}_{T}$ quantified as $\left.\left[q^{3}\left(\operatorname{Pr}_{T}\right)-q^{1}\left(\operatorname{Pr}_{T}\right)\right] / q^{2}\left(\operatorname{Pr}_{T}\right)\right]\left(q^{i}\right.$ means $i$ th quartile) as a function of averaging time $T_{c}$. To contrast unstable and very stable conditions, two stability bins for unstable $(-2.0<\Delta / L$ $<0.0)$ and very stable $(1.5<\Delta / L<5.5)$ atmospheric conditions are selected. The data are from array $1(\Delta / z \sim 4.3)$.

ditions, $\left(q^{3}-q^{1}\right) / q^{2}$ decreases from a value of 1.7 to 0.3 for $T_{c}$ ranging from $T_{c}=0.05 \mathrm{~s}$ to $6.8 \mathrm{~min}$. For very stable conditions, the variability is constant between 0.3 and 0.6 for the entire range of $T_{c}$. This is in agreement with findings for the variability of $c_{s}$ in section 5. Possibly due to the intermittency in stable conditions the variability does not decrease for larger averaging times, while in unstable conditions the variability decreases significantly. The results for arrays 1 , 3 , and 4 are very similar.

\section{Conclusions}

Parameters of the Smagorinsky model for the SGS shear stress and the SGS heat flux have been studied based on a statistical analysis of a large dataset $(157 \mathrm{~h})$ of ABL turbulence. Model coefficients have been measured based on the condition of equivalence between real and modeled SGS dissipation of kinetic energy and scalar variance. Several trends have been identified. Consistent with prior results in the literature, near the ground it is found that $c_{s}$ depends on the ratio of filter length and height above the ground $\Delta / z$ and decreases as $\Delta / z$ is increased. Moreover, $c_{s}$ depends strongly on atmospheric stability as parameterized by the lengthscale ratio $\Delta / L$. The previously postulated decrease of $c_{s}$ in stable stratification and shear (Deardorff 1980; Canuto and Cheng 1997) is quantified from the data, and an empirical formula [Eq. (17)] for $c_{s}$ is proposed. By varying the time $T_{c}$ over which the SGS energy dissipations are averaged, we find that the variability in $c_{s}$ decreases with increasing $T_{c}$ for unstable to neutral conditions, whereas, in very stable conditions, the variability in $c_{s}$ is independent of averaging time. The fact that in either case the median of $c_{s}$ is independent of averaging time confirms the robustness of the results. It also supports the assumption inherent in Lagrangian dynamic SGS models that coefficients can be obtained from data by averaging over timescales that are not overly long.

The dependence of $c_{s}$ on local strain-rate magnitude has also been studied here. Since the Smagorinsky model already assumes proportionality of the eddy viscosity $\nu_{T}$ to strain-rate magnitude $|\tilde{S}|, c_{s}$ should be independent of strain-rate magnitude. The data suggest that this is correct for unstable to neutral conditions or for small strain-rate magnitudes. However, in stable conditions and for large strain-rate magnitudes, $c_{s}$ decreases with strain-rate magnitude. In very stable conditions the data are consistent with a $c_{s}^{2} \sim|\tilde{S}|^{-1}$ scaling. The transition value of the strain-rate magnitude between these two regimes is found to depend on stability and $\Delta / z$. This result shows that the usual velocity scale $l|\tilde{S}|$ is inappropriate under stable conditions, even when correcting the length scale from $\Delta$ to $L$ (i.e., using $l$ ). Instead, the friction velocity provides a better scale for prescribing the eddy viscosity when the turbulence is limited by stable stratification, but one still has to account for the fact that the velocity scale has to be smaller than $u_{*}$ when $\Delta$ is in the inertial range.

A similar analysis is carried out for the coefficient of the SGS heat flux $\operatorname{Pr}_{T}^{-1} C_{s}^{2}$ and the derived turbulent Prandtl number $\operatorname{Pr}_{T}$. The strong decrease of $\operatorname{Pr}_{T}^{-1} c_{s}^{2}$ in stable conditions comes mostly from the strong dependence of $c_{s}^{2}$ on stability, while we observe that $\operatorname{Pr}_{T}$ depends only weakly on stability. A robust increase of $\operatorname{Pr}_{T}$ with increasing $\Delta / z$, going from $\operatorname{Pr}_{T} \sim 0.49$ for $\Delta /$ $z<0.7$, to $\operatorname{Pr}_{T} \sim 0.67$ for $\Delta / z \sim 4.3$, is observed. The observed dependence of the median of $\operatorname{Pr}_{T}$ on the averaging time $T_{c}$ and general variability of the results precludes us from stating unambiguous conclusions on the dependence of $\operatorname{Pr}_{T}$ on stability. In general, results for the SGS heat flux models show more scatter than those for the SGS stress models.

Finally, the basic flaws of the eddy-viscosity models need to be pointed out. Even perfect knowledge of the coefficient does not result in correct prediction of both 
energy transfer from the resolved scales to the subgrid scales and the momentum fluxes associated with the SGS stress. Moreover, the basic proportionality assumption of the Smagorinsky model $\tau_{i j} \propto \Delta^{2}|\tilde{S}| \tilde{S}_{i j}$ is contradicted by tensorial misalignment between SGS stress and strain rate (Tao et al. 2002), independent of the value of $c_{s}$.

Acknowledgments. The authors wish to thank Tom Horst, Donald Lenschow, Chin-Hoh Moeng, Peter Sullivan, and Jeffrey Weil from the NCAR ATD and MMM divisions for this fruitful collaboration during the field experiment. Thanks also to Profs. W. Eichinger, F. PortéAgel, S. Richardson, and J. Wyngaard for the loan of sonic anemometers. The authors gratefully acknowledge funding from the National Science Foundation Grant NSF-ATM 01300766.

\section{APPENDIX}

\section{Test of Filtered Velocity Gradient Accuracy}

In order to assess the data accuracy one can check how closely the measured filtered velocity gradients obey the divergence-free condition:

$$
\frac{\partial \tilde{u}}{\partial x}+\frac{\partial \tilde{v}}{\partial y}+\frac{\partial \tilde{w}}{\partial z}=0 .
$$

Since the equality cannot hold exactly, we must compare the magnitude of the divergence with typical velocity gradient magnitudes. Similar to Zhang et al. (1997) we examine the dimensionless parameter $\eta$ defined according to

$$
\eta=\frac{\left(\frac{\partial \tilde{u}}{\partial x}+\frac{\partial \tilde{v}}{\partial y}+\frac{\partial \tilde{w}}{\partial z}\right)^{2}}{\left(\frac{\partial \tilde{u}}{\partial x}\right)^{2}+\left(\frac{\partial \tilde{v}}{\partial y}\right)^{2}+\left(\frac{\partial \tilde{w}}{\partial z}\right)^{2}} .
$$

This divergence parameter $\eta$ vanishes if the divergence-free condition is obeyed exactly. Moreover, for random data where the individual gradient terms are uncorrelated, $\eta=1$. $\eta$ is bound by $0<\eta<3$. For our data, $\eta$ varies from one data sample to another and so no unique value of $\eta$ exists. Instead, as in Zhang et al. (1997) we measure the probability density function (pdf) of $\eta$ and thus document the frequency of occurrence of different values of $\eta$. Parameter $\eta$ is computed for the four different arrays over the entire dataset and pdfs are plotted in Fig. A1. Clearly $\eta=0$ (satisfaction of continuity) is the most likely value. Between $50 \%$ (for array 1) and $65 \%$ (for array 4) of the data are between $0<\eta<0.5$. Comparing the pdfs with each other one can state that accuracy of gradients decreases with increasing $\Delta / z$. No conclusions can be made about relative errors of $x, y$, or $z$ gradients, but we expect the largest contribution to the error to be from the first-

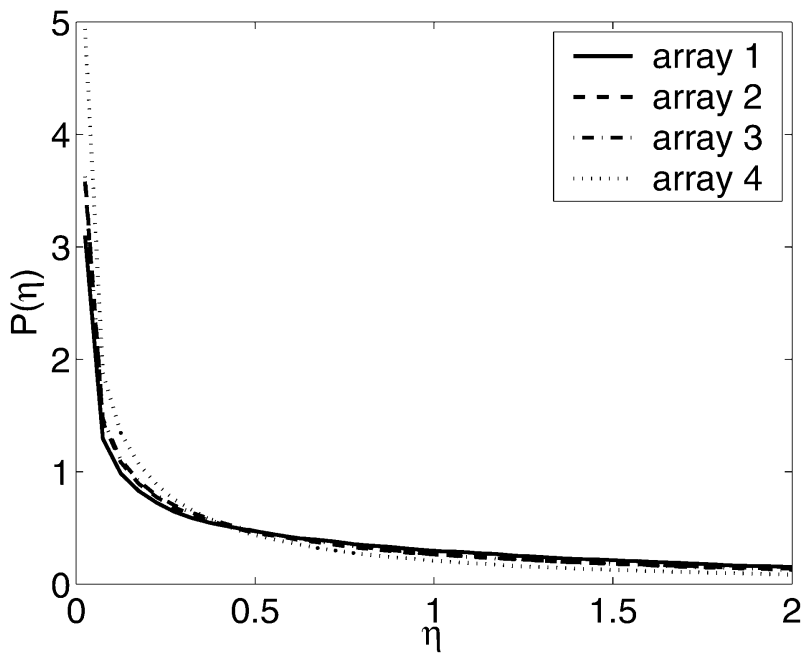

FIG. A1. Probability density distribution of divergence parameter $\eta$ [Eq. (A2)] for the four HATS arrays with different values of $\Delta / z$. Parameter $\eta=0$ for perfect data (satisfying mass continuity), $\eta=$ 1 for pseudorandom data, and $\eta$ is bound by $\eta \leq 3$. All data specified in column 2 of Table 1 is used for the pdf (over $2.5 \times 10^{6}$ data points per array).

order one-sided derivatives in the $z$ direction. The level of error in evaluating derivatives apparent from this test can be considered reasonable (although it is not small).

\section{REFERENCES}

Brown, A. R., P. J. Mason, and S. H. Derbyshire, 1994: Large-eddy simulation of stable atmospheric boundary layers with a revised stochastic subgrid model. Quart. J. Roy. Meteor. Soc., 120, 1485-1512.

Brutsaert, W., 1982: Evaporation into the Atmosphere: Theory, History and Applications. D. Reidel, 299 pp.

Businger, J. A., J. C. Wyngaard, Y. Izumi, and E. F. Bradley, 1971: Flux-profile relationships in the atmospheric boundary layer. $J$. Atmos. Sci., 28, 181-189.

Canuto, V. M., and Y. Cheng, 1997: Determination of the Smagorinsky-Lilly constant $c_{s}$. Phys. Fluids, 7, 1368-1378.

Carati, D., G. Winckelmans, and H. Jeanmart, 2001: On the modelling of the subgrid-scale and filtered-scale stress tensors in largeeddy simulation. J. Fluid Mech., 441, 119-138.

Cerutti, S., and C. Meneveau, 1998: Intermittency and relative scaling of the subgrid dissipation rate in turbulence. Phys. Fluids, $\mathbf{1 0}$ 928-937.

- , and - 2000: Statistics of filtered velocity in grid and wake turbulence. Phys. Fluids, 12, 1143-1165.

- — , and O. M. Knio, 2000: Spectral and hyper eddy viscosity in high-Reynolds-number turbulence. J. Fluid Mech., 421, $307-$ 338.

Clark, R. A., J. H. Ferziger, and W. C. Reynolds, 1979: Evaluation of subgrid models using an accurately simulated turbulent flow. J. Fluid Mech., 91, 1-16.

Deardorff, J. W., 1970: A numerical study of three-dimensional turbulent channel flow at large Reynolds numbers. J. Fluid Mech., 41, 453-480.

, 1971: On the magnitude of the subgrid-scale eddy coefficient. J. Comput. Phys., 7, 120-133.

_ 1980: Stratocumulus-capped mixed layers derived from a three dimensional model. Bound.-Layer Meteor., 18, 495-527.

Germano, M., U. Piomelli, P. Moin, and W. H. Cabot, 1991: A dy- 
namic subgrid-scale eddy viscosity model. Phys. Fluids, 3A, $1760-1765$.

Higgins, C., M. B. Parlange, and C. Meneveau, 2003: Alignment trends of velocity gradients and subgrid scale fluxes in the turbulent atmospheric boundary layer. Bound.-Layer Meteor., in press.

Hunt, J. C. R., D. D. Stretch, and R. E. Britter, 1988: Length scales in stably stratified turbulent flows and their use in turbulence models. Stably Stratified Flow and Dense Gas Dispersion, J.S. Puttock, Ed., Clarendon Press, Oxford, 285-322.

Juneja, A., and J. Brasseur, 1999: Characteristics of subgrid-resolvedscale dynamics in anisotropic turbulence with application to rough-wall boundary layers. Phys. Fluids, 11, 3054-3068.

Kang, H. S., and C. Meneveau, 2002: Universality of large eddy simulation model parameters across a turbulent wake behind a heated cylinder. J. Turbul., 3, paper 032.

Lesieur, M., and O. Metais, 1996: New trends in large-eddy simulations of turbulence. Апnи. Rev. Fluid Mech., 28, 45-82.

Lilly, D. K., 1967: The representation of small-scale turbulence in numerical simulation experiments. Proc. IBM Scientific Computing Symp. on Environmental Sciences, Yorktown Heights, NY, Thomas J. Watson Research Center, 195-209.

Mason, P. J., 1994: Large-eddy simulation: A critical review of the technique. Quart. J. Roy. Meteor. Soc., 120, 1-26.

—_, and S. H. Derbyshire, 1990: Large eddy simulation of the stably-stratified atmospheric boundary layer. Bound.-Layer Meteor., 53, 117-162.

— simulations of boundary layers. J. Fluid Mech., 242, 51-78.

_- and A. R. Brown, 1999: On subgrid models and filter operation in large eddy simulation. J. Atmos. Sci., 56, 2101-2114.

Meneveau, C., 1994: Statistics of turbulence subgrid-scale stresses: Necessary conditions and experimental tests. Phys. Fluids, 6A, 815-833.

_ large-eddy-simulation. Annu. Rev. Fluid Mech., 32, 1-32.

—- T. Lund, and W. Cabot, 1996: A Lagrangian dynamic subgridscale model of turbulence. J. Fluid Mech., 319, 353-385.

Moeng, C.-H., 1984: A large-eddy-simulation model for the study of planetary boundary-layer turbulence. J. Atmos. Sci., 41, 2052 2062.

Moin, P., and J. Kim, 1982: Numerical investigation of channel flow. J. Fluid Mech., 118, 341-377.

— K. Kquires, W. Cabot, and S. Lee, 1991: A dynamic subgridscale model for compressible turbulence and scalar transport. Phys. Fluids, 3A, 2746-2757.
Nieuwstadt, F. T. M., 1984: Some aspects of the turbulent stable boundary-layer. Bound.-Layer Meteor., 30, 31-55.

Piomelli, U., P. Moin, and J. H. Ferziger, 1988: Model consistency in large eddy simulation of turbulent channel flows. Phys. Fluids, 31, 1884-1891.

Pope, S. B., 2000: Turbulent Flows. Cambridge University Press, 971 pp.

Porté-Agel, F., C. Meneveau, and M. B. Parlange, 1998: Some basic properties of the surrogate subgrid-scale heat flux in the atmospheric boundary layer. Bound.-Layer Meteor., 88, 425-444.

$\ldots, \ldots$, and $\_$, 2000a: A scale-dependent dynamic model for large-eddy simulation: Application to a neutral atmospheric boundary layer. J. Fluid Mech., 415, 261-284.

_ M. B. Parlange, C. Meneveau, W. Eichinger, and M. Pahlow, 2000b: Subgrid-scale dissipation in the atmospheric surface layer: Effects of stability and filter dimension. J. Hydrometeor., 1, $75-87$.

—_, M. Pahlow, C. Meneveau, and M. Parlange, 2001a: Atmospheric stability effect on subgrid scale physics of large-eddy simulation. Adv. Water Res., 24, 1085-1102.

- M. B. Parlange, C. Meneveau, and W. E. Eichinger, 2001b: A priori field study of the subgrid-scale heat fluxes and dissipation in the atmospheric surface layer. J. Atmos. Sci., 58, 2673-2698.

Smagorinsky, J., 1963: General circulation experiments with the primitive equations. I. The basic experiment. Mon. Wea. Rev., 91, 99-164.

Sullivan, P., J. McWilliams, and C.-H. Moeng, 1994: A subgrid-scale model for large-eddy simulation of planetary boundary-layer flows. Bound.-Layer Meteor., 71, 247-276.

— T. W. Horst, D. H. Lenschow, C.-H. Moeng, and J. C. Weil, 2003: Structure of subfilter-scale fluxes in the atmospheric surface layer with application to large eddy simulation modeling. J. Fluid Mech., 482, 101-139.

Tao, B., J. Katz, and C. Meneveau, 2002: Statistical geometry of subgrid-scale stresses determined from holographic particle image velocimetry measurements. J. Fluid Mech., 457, 35-78.

Tong, C., J. C. Wyngaard, S. Khanna, and J. G. Brasseur, 1998: Resolvable- and subgrid-scale measurement in the atmospheric surface layer: Technique and issues. J. Atmos. Sci., 55, 31143126.

—,- , and J. G. Brasseur, 1999: Experimental study of the subgrid-scale stresses in the atmospheric surface layer. J. Atmos. Sci., 56, 2277-2292.

Zhang, J., B. Tao, and J. Katz, 1997: Turbulent flow measurement in a square duct with hybrid holographic PIV. Exp. Fluids, 23, 373 381 . 